Aus der Klinik für Nephrologie und Rheumatologie

(Prof. Dr. med. M. Zeisberg)

der Medizinischen Fakultät der Universität Göttingen

\title{
Therapieadhärenz und \\ Arzneimittelwechselwirkungen bei \\ Patienten mit hypertensiver Krise
}

\author{
INAUGURAL-DISSERTATION \\ zur Erlangung des Doktorgrades \\ der Medizinischen Fakultät der \\ Georg-August-Universität zu Göttingen
}

\author{
vorgelegt von \\ Nadine Lach \\ aus \\ Langenhagen
}

Göttingen 2020 
Dekan:

Referent/in:

Ko-Referent/in:

Drittreferent/in:
Prof. Dr. Wolfgang Brück

PD Dr. Manuel Wallbach

Prof. Dr. Christoph Herrmann-Lingen

Prof. Dr. Thomas Meyer

Datum der mündlichen Prüfung: 27.05.2021 
Hiermit erkläre ich, die Dissertation mit dem Titel "Therapieadhärenz und Arzneimittelwechselwirkungen bei Patienten mit hypertensiver Krise" eigenständig angefertigt und keine anderen als die von mir angegebenen Quellen und Hilfsmittel verwendet zu haben.

Göttingen, den

(Unterschrift) 
Die Daten, auf denen die vorliegende Arbeit basiert, wurden teilweise publiziert:

Wallbach M, Lach N, Stock J, Hiller H, Mavropoulou E, Müller GA, Herrmann-Lingen C, Chavanon ML, Neurath H, Blaschke S, Lowin E, Koziolek MJ: Analysis of treatment adherence and drug interactions in patients with hypertensive crisis (Poster). 8. Jahrestagung der Deutschen Gesellschaft für Nephrologie, Berlin, September 2016.

Wallbach M, Lach N, Stock J, Hiller H, Mavropoulou E, Müller GA, Herrmann-Lingen C, Chavanon ML, Neurath H, Blaschke S, Lowin E, Koziolek MJ: Adherence in patients with hypertensive crisis in the emergency department (2. Vortragspreis). 40. Wissenschaftlicher Kongress der Deutschen Hochdruckliga, Berlin, Dezember 2016.

Wallbach M, Lach N, Stock J, Hiller H, Mavropoulou E, Müller GA, Herrmann-Lingen C, Chavanon ML, Neurath H, Blaschke S, Lowin E, Koziolek MJ: Adherence in patients with hypertensive crisis in the emergency department (Posterpreis). 123. Kongress der Deutschen Gesellschaft für Innere Medizin, Mannheim, April 2017.

Wallbach M, Lach N, Stock J, Hiller H, Mavropoulou E, Chavanon ML, Neurath H, Blaschke S, Lowin E, Herrmann-Lingen C et al. (2019): Direct assessment of adherence and drug interactions in patients with hypertensive crisis-A cross-sectional study in the Emergency Department. J Clin Hypertens (Greenwich) 21, 55-63 


\section{Inhaltsverzeichnis}

Abbildungsverzeichnis ................................................................................. III

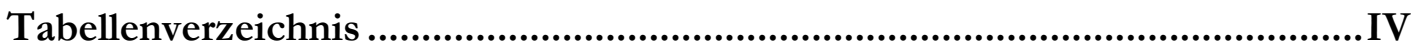

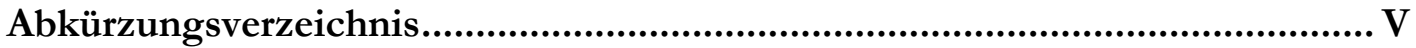

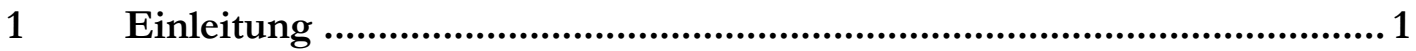

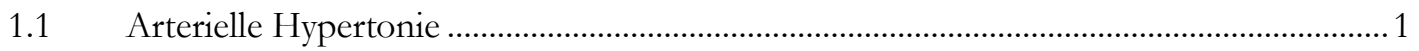

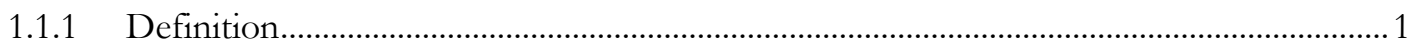

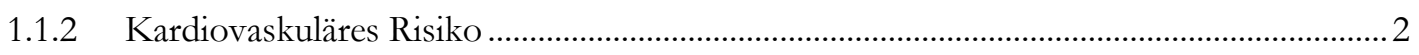

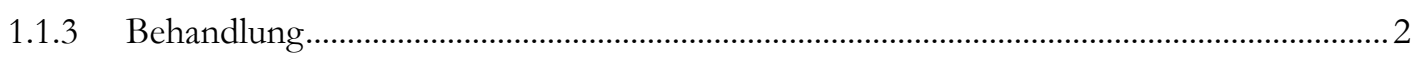

1.2 Hypertensive Krise: Notfall und Dringlichkeit.........................................................

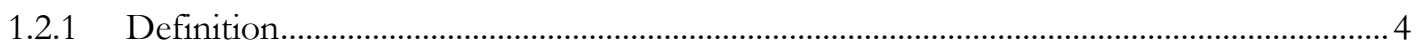

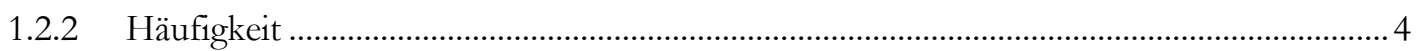

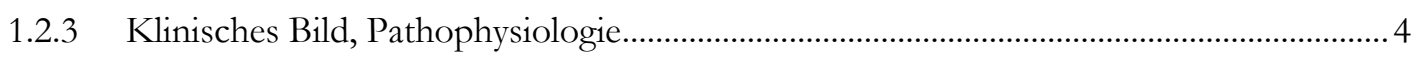

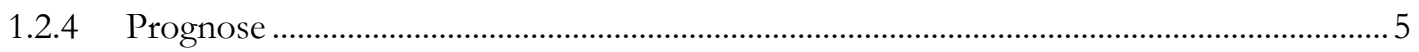

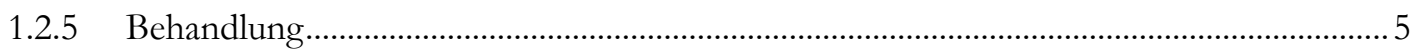

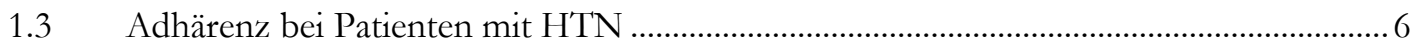

1.3.1 Adhärenz bzw. Non-Adhärenz bei Patienten mit HTN-C ............................................... 8

1.4 Blutdrucksteigernde Arzneimittel und Stoffe ……………..............................................

$1.5 \quad$ Fragestellung ……………………………………………………………………….... 10

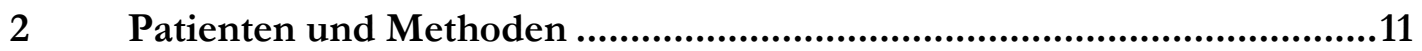

2.1 Votum der Ethikkommission ........................................................................................11

2.2 Aufnahme in die Studie _........................................................................................11

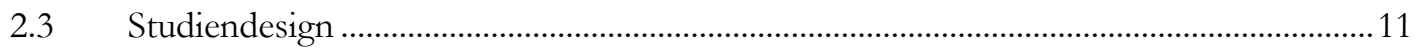

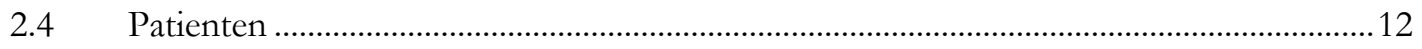

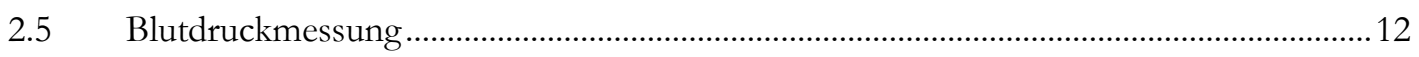

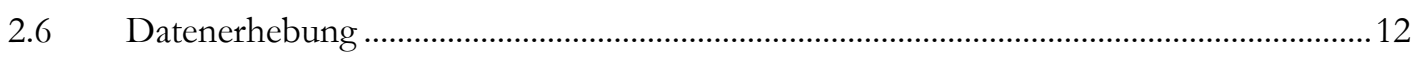

2.6.1 Gemeinsam erhobene Parameter............................................................................13

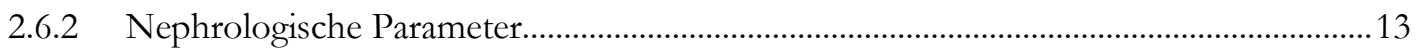

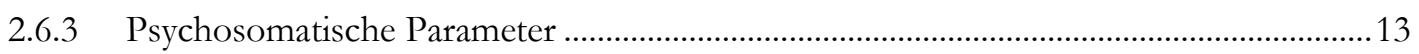

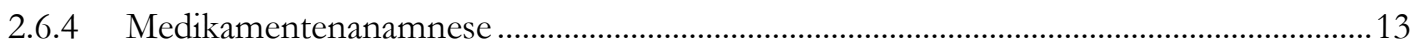

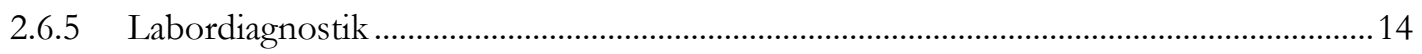

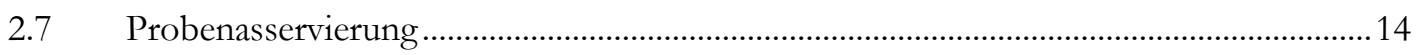

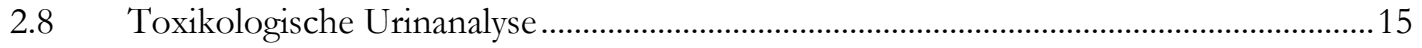

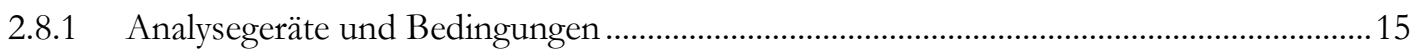

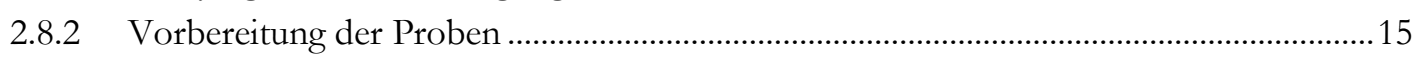

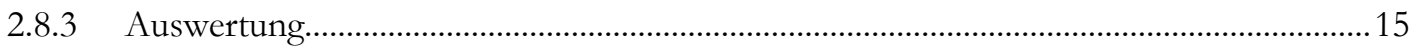

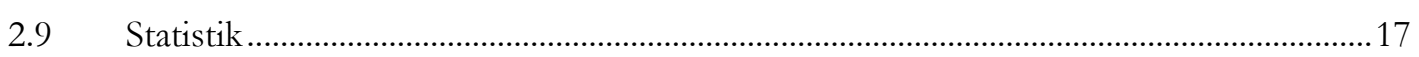

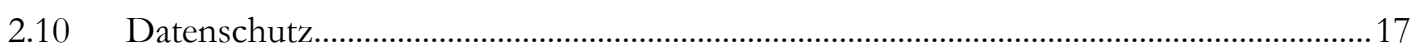




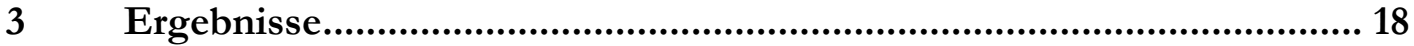

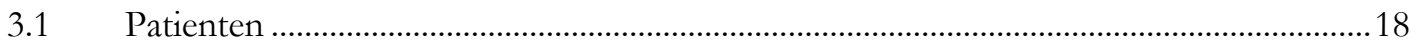

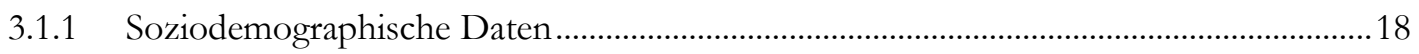

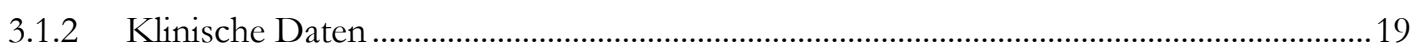

3.1.3 Antihypertensive Medikation..................................................................................22

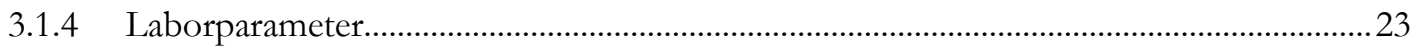

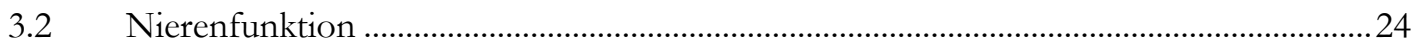

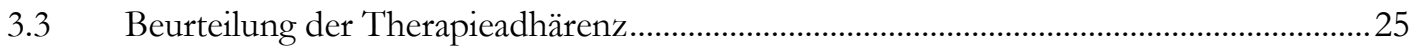

3.3.1 Adhärenz in Bezug auf die Substanzklasse...............................................................27

3.4 Vergleich zwischen adhärenten und non-adhärenten Patienten.......................................28

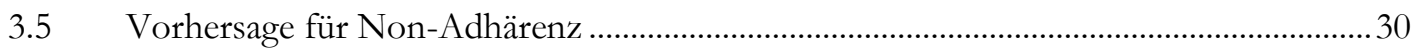

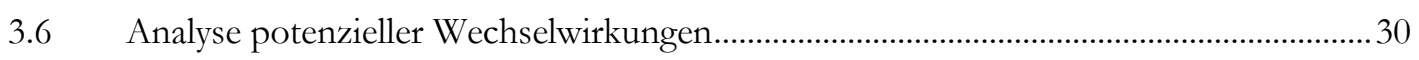

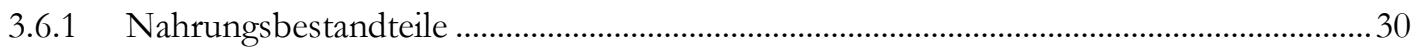

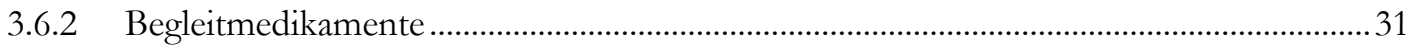

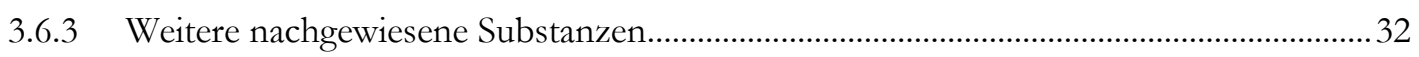

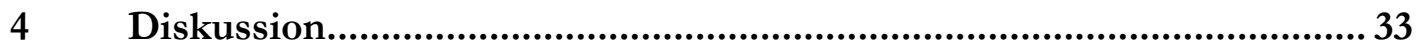

4.1 Der prozentuale Anteil an non-adhärenten Patienten...........................................................33

4.2 Stärken und Schwächen der direkten Adhärenzmessung bei Patienten mit HTN-C....34

4.3 Prädiktoren, die auf das Vorliegen einer Non-Adhärenz in diesem Kollektiv

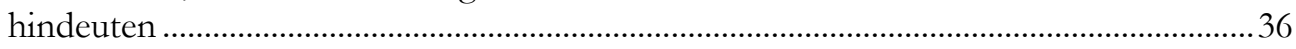

4.4 Hoher Anteil an potenziell blutdrucksteigernden Medikamenten bzw. Nahrungsmitteln bei Patienten mit HTN-C ......................................................................37

4.5 Methodendiskussion ...................................................................................................

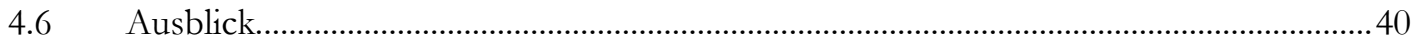

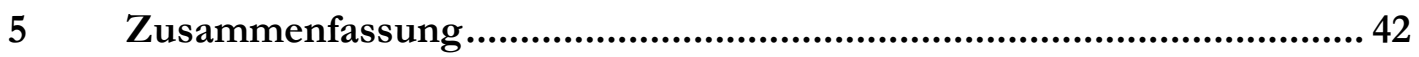

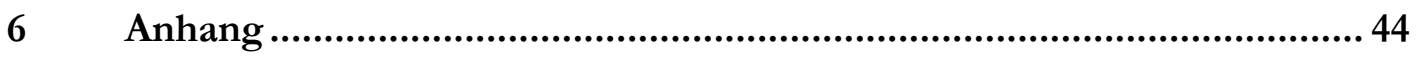

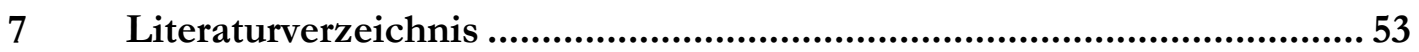




\section{Abbildungsverzeichnis}

Abbildung 1: Flowchart der Studie.

Abbildung 2: Symptome bei Vorstellung in der Notaufnahme.

Abbildung 3: Verteilung der Patienten auf die Stadien G1-G5 der chronischen

Nierenerkrankung (chronic kidney disease; CKD), ...............................................................2

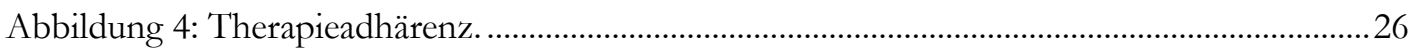

Abbildung 5: Verteilung des Adhärenzgrades..........................................................................2

Abbildung 6: Therapieadhärenz in den verschiedenen Substanzklassen......................................2

Abbildung 7: Einnahme bestimmter Nahrungsmittel. ...................................................................

Abbildung 8: Einnahme verschriebener Antidepressiva..................................................................

Abbildung 9: Einnahme nichtsteroidaler Antirheumatika insgesamt...............................................32 


\section{Tabellenverzeichnis}

Tabelle 1: Definition und Klassifikation der HTN...................................................................... 1

Tabelle 2: Ein- und Ausschlusskriterien der Studie. ....................................................................... 12

Tabelle 3: Strukturierte Abfrage der antihypertensiven Medikation. .............................................14

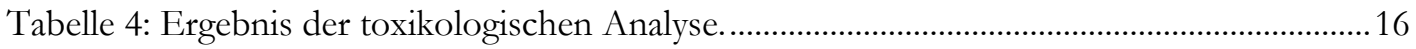

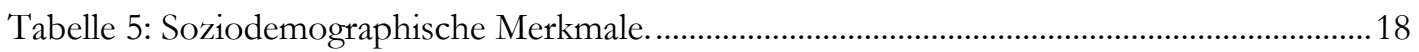

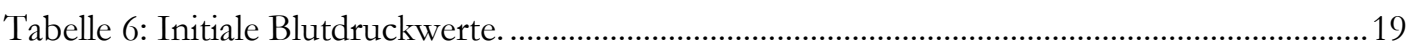

Tabelle 7: Manifestationen von Endorganschäden und deren Häufigkeit bei hypertensiven

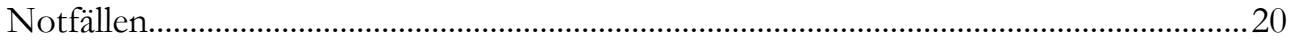

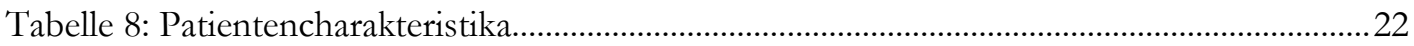

Tabelle 9: Antihypertensive Substanzklassen............................................................................23

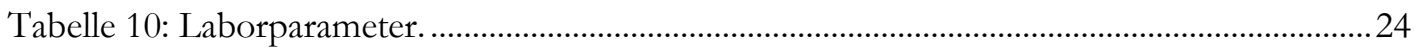

Tabelle 11: Geschätzte glomeruläre Filtrationsrate......................................................................24

Tabelle 12: Vergleich zwischen adhärenten und non-adhärenten Patienten. ..............................29

Tabelle 13: Spearmans Rangkorrelation zwischen Glucose und Blutdruck.................................30 


\section{Abkürzungsverzeichnis}

ACE angiotensin converting ensyme, Angiotensin-konvertierendes Enzym

BMI Body-Mass-Index

CRP

C-reaktives Protein

eGFR estimated glomerular filtration rate, geschätzte glomeruläre Filtrationsrate

ESC European Society of Cardiology

$\mathrm{ESH}$ European Society of Hypertension

GC-MS Gas-chromatography Mass Spectrometry, Gaschromatographie-

HTN Massenspektrometrie

HTN-C arterielle Hypertonie

IQR hypertensive Krise

LC-MS interquartile range, Interquartilsabstand

Liquid-chromatography Mass Spectrometry, FlüssigchromatographieMassenspektrometrie

NSAID non-steroidal anti-inflammatory drug, nichtsteroidales Antirheumatikum

RR

Riva-Rocci

SD

Standardabweichung

TSH

Thyreoidea-stimulierendes Hormon

WHO

World Health Organization 


\section{$1 \quad$ Einleitung}

\subsection{Arterielle Hypertonie}

Die arterielle Hypertonie (HTN) ist die häufigste chronische Erkrankung in der westlichen Welt. In Deutschland waren in den Jahren 2008-2011 rund 32\% der Erwachsenen im Alter von 18 bis 79 Jahren von einer HTN betroffen. Die Anzahl der Betroffenen steigt mit dem Alter stetig an und erreicht in der Altersgruppe der 65 bis 79-Jährigen sogar mehr als 70\% (Neuhauser et al. 2015).

\subsubsection{Definition}

Laut den gemeinsamen Empfehlungen der European Society of Cardiology (ESC) und der European Society of Hypertension (ESH) ist eine HTN definiert als eine Erhöhung des systolischen Praxis-Blutdruckwertes auf $\geq 140 \mathrm{mmHg}$ und/oder des diastolischen Blutdruckwertes auf $\geq 90 \mathrm{mmHg}$ (Williams et al. 2018). Die Einteilung für Erwachsene in verschiedene Schweregrade ist in Tabelle 1 dargestellt.

Tabelle 1: Definition und Klassifikation der HTN (Williams et al. 2018).

\begin{tabular}{|l|c|c|}
\hline Kategorie & Systolisch (mmHg) & Diastolisch (mmHg) \\
\hline Optimal & $<120$ & $<80$ \\
\hline Normal & $120-129$ & $80-84$ \\
\hline Hochnormal & $130-139$ & $90-99$ \\
\hline Grad I Hypertonie & $140-159$ & $100-109$ \\
\hline Grad II Hypertonie & $160-179$ & $\geq 110$ \\
\hline Grad III Hypertonie & $\geq 180$ & 29 \\
\hline
\end{tabular}

Entsprechend wird ein systolischer Blutdruck von 130-139 $\mathrm{mmHg}$ und/oder ein diastolischer Blutdruck von 85-89 mmHg als hochnormal klassifiziert. Die Diagnose „HTN“ sollte auf Blutdruckmessungen bei wiederholten Praxisbesuchen beruhen. Ein Praxisbesuch 
sollte drei Blutdruckmessungen in 1-2 Minuten Abstand beinhalten. Der Blutdruck wird als Durchschnitt der letzten beiden Messungen aufgezeichnet. Die Kategorie wird durch den jeweils höchsten Blutdruckwert bestimmt, sei er systolisch oder diastolisch (Williams et al. 2018).

Eine weitere Einteilung basiert auf der Ätiologie der HTN. Kann den erhöhten Blutdruckwerten keine Ursache zu Grunde gelegt werden, spricht man von einer primären HTN und meint eine multifaktorielle, polygene Erkrankung (Herold 2018). Entsteht der hohe Blutdruck hingegen als Symptom einer anderen Grunderkrankung bzw. durch nachweisbare Krankheitsursachen wird die Form der HTN als sekundär bezeichnet.

\subsubsection{Kardiovaskuläres Risiko}

Obwohl die HTN meist ohne Beschwerden beginnt, führt die Erhöhung der Blutdruckwerte durch die hämodynamische Belastung zu Organschädigungen. Das Risiko von Patienten mit Bluthochdruck einen Herzinfarkt, eine Herzinsuffizienz, einen Schlaganfall oder ein Nierenversagen $\mathrm{zu}$ erleiden, ist im Vergleich $\mathrm{zu}$ nicht Betroffenen bedeutend erhöht (Chobanian et al. 2003). Zusätzlich wird eine HTN oft von begünstigenden Faktoren wie Dyslipidämie, Glukoseintoleranz, Fettleibigkeit und linksventrikulärer Hypertrophie begleitet, die in Kombination mit dem hohen Blutdruck die kardiovaskuläre Morbidität und Mortalität verstärken (Kannel 1996). Die Erhebungen der World Health Organization (WHO) 2002 zeigen, dass etwa 62\% der cerebrovaskulären Erkrankungen und 49\% der koronaren Herzerkrankungen auf einen suboptimalen Blutdruck (systolisch $>115 \mathrm{mmHg}$ ) zurückzuführen sind. Steigende Blutdruckwerte $(\geq 115 / 75 \mathrm{mmHg})$ stehen in linearem Zusammenhang mit einem Anstieg der kardiovaskulären Mortalität. Jede Steigerung um $20 \mathrm{mmHg}$ systolisch oder $10 \mathrm{mmHg}$ diastolisch verdoppelt das Risiko, an einem Schlaganfall, einer koronaren Herzkrankheit oder einer anderen vaskulären Ursache zu versterben (Lewington et al. 2002). Weltweit werden jährlich 7,1 Millionen Todesfälle durch erhöhten Blutdruck verursacht (World Health Organization 2002).

\subsubsection{Behandlung}

Die Behandlung der HTN basiert auf den folgenden drei Säulen: (1) Lebensstiländerungen, (2) Medikamentöse Therapie und (3) Gerätebasierte Therapie, die hauptsächlich gezielt zur Behandlung von resistenter HTN eingesetzt werden sollte.

Lebensstiländerungen werden für alle Patienten mit hochnormalem Blutdruck oder HTN empfohlen. Eine gesunde Lebensweise kann das Auftreten einer HTN verhindern oder 
verzögern und das kardiovaskuläre Risiko reduzieren. Effektive Änderungen im Lebensstil können ausreichen, um die Notwendigkeit einer medikamentösen Therapie bei Patienten mit HTN Grad I zu verzögern oder zu verhindern. Sie können auch die Wirkung einer blutdrucksenkenden Therapie bei behandelten Patienten verstärken, aber sie sollten niemals den Beginn einer Pharmakotherapie bei Patienten mit einem durch die HTN bedingten Organschaden oder einem hohen kardiovaskulären Risiko verzögern. Empfohlene Lebensstil-Interventionen, für die ein blutdrucksenkender Effekt belegt ist, sind Salzrestriktion, Einschränkung des Alkoholkonsums, hoher Konsum von Gemüse und Früchten, Gewichtsnormalisierung und regelmäßige körperliche Aktivität. Darüber hinaus sollten hypertensive Raucher beraten werden, das Rauchen zu beenden (Williams et al. 2018).

Die meisten HTN-Patienten benötigen zusätzlich $\mathrm{zu}$ Lebensstiländerungen eine medikamentöse Therapie, um eine optimale Blutdruckkontrolle zu erreichen. Das erste Ziel der medikamentösen Behandlung besteht laut den aktuellen ESC/ESH Leitlinien (2018) darin, den Blutdruck bei allen Patienten auf $<140 / 90 \mathrm{mmHg}$ zu senken und, sofern die Therapie gut vertragen wird, sollten bei den meisten Patienten unter Behandlung Blutdruckwerte von 130/80 mmHg oder niedriger angestrebt werden. Ausnahmen gelten für ältere Patienten ( $\geq 65$ Jahre) und/oder Patienten mit chronischer Niereninsuffizienz, bei denen ein systolischer Blutdruck im Bereich von 130-139 mmHg empfohlen wird. Basis der blutdrucksenkenden Therapie sind die vier Hauptsubstanzklassen angiotensin converting ensyme (ACE)-Hemmer, Angiotensin-II-Blocker, Kalziumantagonisten und Diuretika. Jede dieser Substanzklassen hat absolute oder relative Kontraindikationen gegen ihren Einsatz. Bei unkomplizierter HTN wird empfohlen mit einer Zwei-Wirkstoff-Kombination bevorzugt aus einem Hemmstoff des Renin-Angiotensin-Systems (entweder ACE-Hemmer oder Angiotensin-II-Blocker) und einem Kalziumantagonisten oder einem Diuretikum zu beginnen. Bei Niedrigrisiko-Patienten mit HTN Grad I, deren systolischer Blutdruck $<150 \mathrm{mmHg}$ ist, kann auch eine Monotherapie als Initialtherapie genutzt werden. Der Einsatz eines Betablockers wird bei Patienten mit HTN und koronarer Herzkrankheit oder HTN und Herzinsuffizienz in Kombination mit einer der vorgenannten Hauptsubstanzklassen empfohlen. Lässt der Blutdruck sich mit einer Zwei-WirkstoffKombination nicht einstellen, sollte die Therapie auf eine Drei-Wirkstoff-Kombination erhöht werden: in der Regel ein Hemmstoff des Renin-Angiotensin-Systems, ein Kalziumantagonist und ein Diuretikum (Williams et al. 2018). Wird der Zielblutdruck trotz regelmäßiger Einnahme einer Drei-Wirkstoff-Kombination (einschließlich Diuretikum) in optimaler Dosis nicht erreicht, gilt die HTN als therapieresistent (Chobanian et al. 2003), 
und die aktuellen Leitlinien empfehlen, die Hinzugabe von Spironolacton oder anderen langwirksamen Diuretika, einem Betablocker oder einem Alphablocker (Williams et al. 2018).

Bei der Behandlung mit einer Kombination sollten bevorzugt zwei oder mehr Wirkstoffe als Einzeltablette verwendet werden. Eine Verringerung der täglichen Tablettenzahl verbessert die häufig niedrige Einnahmetreue der Patienten und erhöht die Geschwindigkeit der Blutdruckkontrolle (Corrao et al. 2010; Gupta et al. 2010). Untersuchungen an sehr großen HTN-Kohorten haben gezeigt, dass die initiale Kombinationstherapie weniger Behandlungsabbrüche und ein geringeres Risiko für kardiovaskuläre Ereignisse zur Folge hat (Corrao et al. 2011; Corrao et al. 2010).

\subsection{Hypertensive Krise: Notfall und Dringlichkeit}

\subsubsection{Definition}

Ein hypertensiver Notfall ist definiert als deutliche kritische Erhöhung des Blutdruckes $(>180 / 120 \mathrm{mmHg})$, verbunden mit einer drohenden oder progressiven Endorganschädigung (Chobanian et al. 2003). Dabei ist zu beachten, dass die Definition in der Literatur nicht einheitlich gehandhabt wird und auch andere Grenzwerte beschrieben sind. Wichtig scheint weniger der absolute Blutdruckwert als vielmehr der relative und plötzliche Blutdruckanstieg zu sein und der nachweisbare Organschaden. Davon zu trennen ist eine deutliche Blutdruckerhöhung ohne akuten Organschaden bzw. ohne ausgeprägte Symptome (hypertensive Dringlichkeit).

\subsubsection{Häufigkeit}

HTN macht bei Erwachsenen bis zu 0,9\% aller Besuche in der Notaufnahme aus (McNaughton et al. 2015), Hypertensive Krise (HTN-C) 0,5\% (Pinna et al. 2014) davon ca. 0,2\% bei hypertensivem Notfall (Janke et al. 2016). In der HTN-Population entwickeln ungefähr 1-2\% der Patienten irgendwann einmal eine HTN-C (Varounis et al. 2016). Eine HTN-C kann auch bei Patienten mit zuvor unbekannter HTN auftreten als erste Präsentation ihres hypertensiven Zustandes (Pinna et al. 2014).

\subsubsection{Klinisches Bild, Pathophysiologie}

Krisenhafte Blutdruckanstiege gehören zu den Komplikationen einer HTN. Im Falle eines hypertensiven Notfalls können sie unbehandelt zu einem irreversiblen Organschaden oder -versagen führen und dadurch das Leben des Patienten akut bedrohen. Klassischerweise 
finden sich beim hypertensiven Notfall deutliche neurologische Veränderungen (cerebraler Insult, cerebrale Blutung, hypertensive Enzephalopathie) oder andere Organmanifestationen wie akutes Linksherzversagen mit (Prä-)Lungenödem, akutes Koronarsyndrom, Eklampsie oder Aortendissektion. Die häufigsten Symptome der Patienten sind Kopfschmerzen, Schwindel, Nasenbluten, Benommenheit und psychomotorische Agitation bei hypertensiven Dringlichkeiten und Brustschmerzen, Dyspnoe und neurologische Defizite bei hypertensiven Notfällen (Martin et al. 2004; Zampaglione et al. 1996).

Die Pathophysiologie der HTN-C ist komplex und bis heute nicht vollkommen geklärt. Zwei unterschiedliche, aber miteinander verbundene Mechanismen können eine zentrale Rolle spielen. Erstens ein Versagen der Autoregulationsmechanismen im Gefäßbett und zweitens die Aktivierung des Renin-Angiotensin-Systems, die eine weitere Vasokonstriktion bewirkt. So entsteht ein Circulus vitiosus aus fortwährenden endothelialen Verletzungen und anschließender Ischämie (Varounis et al. 2016).

\subsubsection{Prognose}

Bei Patienten mit hypertensivem Notfall ist die Mortalität höher (4,6\%) als bei Patienten mit hypertensiven Dringlichkeiten (0,8\%) (Gonzalez Pacheco et al. 2013) mit einer 30-TageMortalität von 4\% bei Patienten, die eine parenterale antihypertensive Medikation zur Behandlung der HTN-C benötigen (Vuylsteke et al. 2011). Bei 29\% der aufgrund einer schweren HTN stationär behandelten Patienten wird innerhalb von 90 Tagen nach der Klinikentlassung ein weiterer durch die HTN bedingter Krankenhausaufenthalt notwendig (Gore et al. 2010).

\subsubsection{Behandlung}

Patienten mit hypertensivem Notfall sollten zur kontinuierlichen Überwachung von Blutdruck und Endorganschaden und zur parenteralen Gabe eines geeigneten Arzneimittels auf einer Intensivstation (Intensive Care Unit) aufgenommen werden. Wie stark der Blutdruck gesenkt wird, hängt von der jeweiligen Erkrankung ab. Für Erwachsene mit einer zwingenden Erkrankung (d. h. Aortendissektion, schwere Präeklampsie oder Eklampsie oder Phäochromozytomkrise) wird eine Senkung des systolischen Blutdruckes auf $<140 \mathrm{mmHg}$ während der ersten Stunde empfohlen und auf $<120 \mathrm{mmHg}$ bei Aortendissektion. Für Erwachsene ohne zwingende Erkrankung sollte der systolische Blutdruck um 25\% des Ausgangswertes während der ersten Stunde reduziert werden und, sofern stabil, auf 160/100$110 \mathrm{mmHg}$ während der nächsten 2 bis 6 Stunden. In den folgenden 24 bis 48 Stunden sollte dann vorsichtig ein normaler Blutdruckwert erreicht werden. Die klinische Erfahrung zeigt, 
dass eine zu starke Blutdrucksenkung zu Nieren-, Hirn- oder Koronar-Ischämie führen oder dazu beitragen kann und sollte vermieden werden (Whelton et al. 2018).

Hoher Blutdruck, der eine parenterale Therapie erfordert, wird mit einer Vielzahl von Arzneimittelwirkstoffen in Europa behandelt, am häufigsten werden Nitroglycerin, Urapidil und Clonidin verwendet (Vuylsteke et al. 2011).

Im Gegensatz dazu ist bei der hypertensiven Dringlichkeit keine Überweisung an die Notaufnahme, sofortige Senkung des Blutdruckes in der Notaufnahme oder Krankenhauseinweisung indiziert. Die Patienten sollten stattdessen durch Wiedereinsetzen oder Intensivierung der oralen blutdrucksenkenden medikamentösen Therapie behandelt werden und gegebenenfalls der Behandlung von Angstzuständen. Wichtig ist auch ein gesichertes Follow-up (Whelton et al. 2018). Viele dieser Patienten haben die blutdrucksenkende Therapie im Vorfeld beendet oder nicht wie mit dem Arzt gemeinsam vereinbart eingehalten (Whelton et al. 2018). Absetzen bestimmter Blutdruckmedikamente, insbesondere Medikamente, die auf das sympathische Nervensystem wirken, kann einen plötzlichen Anstieg des Blutdruckes verursachen, der als Rebound-Hypertonie bezeichnet wird (Ipek et al. 2017). Darüber hinaus kann die Einnahme einer Vielzahl von therapeutischen oder chemischen Substanzen entweder per se zu einer Erhöhung des Blutdruckes führen oder die Wirkung von Antihypertensiva dementsprechend beeinflussen, dass es zu krisenhaften Blutdruckanstiegen kommen kann (Grossman et al. 2015; Grossman und Messerli 2008, 2012).

\subsection{Adhärenz bei Patienten mit HTN}

„Der Begriff Adhärenz beschreibt das Ausmaß, in dem das Verhalten einer Person wie die Einnahme eines Medikamentes, ein Diätregime und/oder eine Lebensstiländerung mit den Empfehlungen übereinstimmt, die mit dem Arzt oder Therapeuten gemeinsam vereinbart wurden (Sabaté 2003).“

Es ist bekannt, dass Non-Adhärenz ein wichtiges Hindernis für den Behandlungserfolg vieler chronischer Erkrankungen, einschließlich HTN, darstellt. Laut eines Berichtes der WHO liegt die Non-Adhärenz von Patienten mit chronischen Krankheiten in den Industrieländern bei ca. 50\% (Sabaté 2003).

Non-Adhärenz kann nach den verschiedenen Ursachen unterteilt werden in (Schönborn 2009): (1) Adaptive Non-Adhärenz: Der Patient passt die ihm verschriebene Therapie seinem Leben und den aktuellen Lebensumständen an, setzt Medikamente entweder sofort oder 
später ab, weil ihm seine Lebensqualität ohne Therapie höher erscheint als mit der empfohlenen Therapiemaßnahme; (2) Beanspruchungs-Non-Adhärenz: Der Patient führt die Therapie nicht oder nicht weiter durch, weil er dazu beispielsweise durch die Komplexität des Therapieplanes nicht in der Lage ist; (3) Intelligente Non-Adhärenz: Der Patient führt ganz bewusst eine bestimmte Therapie nicht oder nicht weiter durch, weil unerwünschte Wirkungen aufgetreten sind oder die Ansicht besteht, dass die Therapie nicht den erwarteten Erfolg bringe.

Trotz der Vielfalt und Verfügbarkeit von wirksamen Medikamenten zur Behandlung einer HTN, hat nur rund die Hälfte der erwachsenen HTN-Patienten ihren Blutdruck ausreichend kontrolliert (Neuhauser et al. 2015). Ein Faktor, der wesentlich dazu beiträgt und Studien und Forschung motiviert ist die medikamentöse Non-Adhärenz. Mehrere Untersuchungen belegen, dass ungefähr die Hälfte der HTN-Patienten während des ersten Jahres die Einnahme ihrer neu verschriebenen Medikamente beendet und eine beträchtliche Anzahl derer, die ihre Medikamente weiterhin einnehmen dies nicht kontinuierlich bzw. nicht nach Dosierungsplan tun (Van Wijk et al. 2005; Vrijens et al. 2008). Die von Patienten am meisten genannten Gründe deuten auf Schwächen einer angemesseneren Kommunikation zwischen dem Patienten und seinem Arzt in Bezug auf die Krankheit und die schwerwiegenden Folgen eines Behandlungsabbruchs hin. Auftreten von unerwünschten Arzneimittelwirkungen war die zweite Ursache für die Aufgabe der Behandlung (Andrade et al. 2002).

Die tatsächliche Prävalenz von Non-Adhärenz bei Patienten mit HTN ist nicht bekannt. Schätzungen variieren stark wegen der Inkonsistenz der verschiedenen Studiendesigns und des Mangels an direkten und objektiven Methoden zur Beurteilung. Die Wahl einer geeigneten Methode zur Beurteilung der Adhärenz hängt von mehreren Faktoren ab, einschließlich Zuverlässigkeit, Empfindlichkeit, Bildungswert, örtliche Einrichtungen, langfristige Durchführbarkeit, Patientenprofil und finanzielle Ressourcen. Indirekte Methoden sind einfach, kostengünstig und zeiteffizient und sie implizieren eine angemessene Arbeitsbelastung. Beispiele für indirekte Methoden sind die Einschätzung durch den Arzt oder das Pflegepersonal, Selbstberichte des Patienten (Interviews/Fragebögen) und die Messung von therapeutischen Wirkparametern oder des Arzneimittelverbrauchs im Verhältnis zur Verschreibung. Wegen der schlechten Empfindlichkeit sind indirekte Methoden stark abhängig vom Verhalten des Patienten, beeinflusst durch soziale Erwünschtheit und Erinnerungsverzerrung (Berra et al. 2016). Insbesondere in einer Stichprobe von 47 Patienten mit scheinbar resistenter HTN wurde geringe Adhärenz auf der Morisky Medication Adherence Scale-8 (26\%) grob unterschätzt im Vergleich zur objektiv 
erfassten Prävalenz von Non-Adhärenz mittels Arzneimittelüberwachung im Blut (51\%) (Pandey et al. 2015). Direkte Methoden sind genauer und zuverlässiger als indirekte Methoden, aber auch teurer und anspruchsvoller im Hinblick auf personelle Ressourcen. Beispiele für direkte Methoden sind die beobachtete Medikamenteneinnahme, elektronische Beobachtungssysteme wie das "Medication Event Monitoring System" (MEMS) und Telemonitoring (Berra et al. 2016). Die Messung des Arzneimittel- bzw. Metaboliten-Spiegels in Körperflüssigkeiten ist die genaueste objektive Methode. Das meistverwendete Probenmaterial bei HTN-Patienten sind Blut und Urin (Berra et al. 2016). Damit wird eine leistungsstarke Chromatographie gekoppelt mit einem empfindlichen Detektor wie Massenspektrometrie als analytische Referenztechnik betrachtet (Berra et al. 2016; Maurer 2007) (Maurer et al. 2017).

In einer ersten Studie mit 76 therapieresistenten hypertensiven Patienten konnte durch Flüssigchromatographie-Massenspektrometrie (LC-MS)-Analyse der blutdrucksenkenden Medikamente oder ihrer Metaboliten im Urin eine Non-Adhärenz-Rate von 53\% gemessen werden (Jung et al. 2013). Eine Untersuchung der Adhärenz an Patienten in einem Hypertonie-Zentrum mittels Hochleistungsflüssigchromatographie Tandem Massenspektrometrie (HP LC-MS/MS) zeigte, dass insgesamt 25\% der Patienten vollständig oder teilweise non-adhärent waren (Tomaszewski et al. 2014).

Schmieder et al. (2016) stellten die tatsächliche Einnahme aller blutdrucksenkenden Medikamente zu Studienbeginn und sechs Monate nach der renalen Denervation (durch LCMS) bei 56\% bzw. 66\% der Patienten mit therapieresistenter HTN fest (Schmieder et al. 2016).

\subsubsection{Adhärenz bzw. Non-Adhärenz bei Patienten mit HTN-C}

In einer prospektiven Längsschnittstudie wurden mehrere potenzielle Risikofaktoren für das Auftreten einer HTN-C identifiziert, einschließlich weibliches Geschlecht, Grad der Fettleibigkeit, hypertensive oder koronare Herzkrankheit, somatoforme Störung und eine höhere Anzahl der blutdrucksenkenden Medikamente (Saguner et al. 2010). Non-Adhärenz war der eindeutig wichtigste Risikofaktor. Wu et al. (2010) zeigten an einer retrospektiven Kohortenbeobachtung, dass geringe Adhärenz mit einer schlechten Blutdruckkontrolle und einem erhöhten Risiko für kardiovaskuläre Erkrankungen und Krankenhausaufenthalte einhergeht. Bender et al. überprüften 2006 die Daten von 50 Notfallpatienten mit hypertensiver Dringlichkeit: In 12\% der Fälle wurde Non-Adhärenz festgestellt. Weitere 16\% der Patienten nahmen ihre Medikamente nicht, weil sie diese vor kurzem aufgebraucht 
hatten. Die Ergebnisse deuten darauf hin, dass mehr als ein Viertel der Besuche hätte verhindert werden können, wären die Patienten tatsächlich unter Therapie gewesen (Bender et al. 2006).

Die bisherigen Studien zur Adhärenz bzw. Non-Adhärenz bei Patienten mit HTN-C weisen wesentliche Einschränkungen, wie retrospektives Design oder Verwendung von ausschließlich indirekten Methoden zur Adhärenzbestimmung (z. B. Fragebögen, klinische Einschätzung oder Auswertung von Rezepteinlösungen) auf. Adhärenzdaten aus direkten Adhärenzmessungen mittels chemisch-toxikologischer Verfahren in dieser Patientengruppe fehlen bis dato vollständig.

\subsection{Blutdrucksteigernde Arzneimittel und Stoffe}

Eine Vielzahl von Arzneimitteln sowie Nahrungs-/Genussmittel bzw. Suchtstoffe können blutdrucksteigernde Effekte vermitteln. Einige Mittel wie z. B. Glucocorticoide, nichtsteroidale Antirheumatika (NSAIDs) verursachen entweder eine Natriumretention und eine extrazelluläre Volumenexpansion oder aktivieren direkt oder indirekt das sympathische Nervensystem (Coffein). Andere Substanzen wie z. B. Cyclosporin A, Tacrolimus wirken direkt auf die glatte Muskulatur der Arteriolen oder haben keinen definierten Wirkmechanismus (Grossman und Messerli 2008, 2012). Arzneimittelinduzierte Blutdruckanstiege sind in der Regel gering und vorübergehend, obwohl seltene hypertensive Notfälle im Zusammenhang mit der Einnahme bestimmter Medikamente gemeldet wurden (Grossman et al. 2015; Grossman und Messerli 2008, 2012). Wichtige internistische Medikamente, die eine HTN auslösen bzw. verstärken können, sind Steroidhormone, NSAIDs, Erythropoietin, Cyclosporin A, Tacrolimus, Alkylanzien, Vascular Endothelial Growth Factor (VEGF)-Hemmer, Sympathomimetika (z. B. in Nasentropfen), daneben Genussgifte wie Nikotin, Alkohol oder Coffein und illegale Drogen wie Amphetamine, „Ecstasy“ oder Kokain. Gemäß den Fachinformationen weisen auch viele Psychopharmaka ein HTN-Risiko auf. Vereinzelt zeigten sich Gruppeneffekte wie z. B. Blutdruckerhöhung durch noradrenerge Antidepressiva wie Selektive Serotonin Noradrenalin Reuptake Inhibitoren (SSNRI) oder auch Stimulanzien (Freudenmann et al. 2017).

Mehrere Fallberichte über HTN-C wurden nach Behandlung mit NSAIDs veröffentlicht. Zwei Studien konnten eine Steigerung des Blutdruckes von $+10 /+7 \mathrm{mmHg}$ bzw. +12/+5 mmHg nach der Hinzugabe von NSAIDs zur blutdrucksenkenden Therapie nachweisen. Bei Patienten mit bereits bestehender HTN ist die Höhe des Blutdruckanstiegs im Vergleich zu normotensiven Personen weniger vorhersehbar und kann mit dem Alter, 
dem Ausgangsblutdruck, der Art des NSAIDs und einer gleichzeitigen blutdrucksenkenden Behandlung variieren. Insbesondere salzempfindliche Patienten oder Patienten mit niedriger Plasma-Renin-Aktivität (z. B. ältere Patienten, Diabetiker und Patienten mit eingeschränkter Nierenfunktion) weisen unter NSAID-Einnahme ein höheres Risiko auf eine schwere HTN zu entwickeln (Wilson und Poulter 2006).

\subsection{Fragestellung}

In der Literatur wurden mehrere potenzielle Risikofaktoren für das Auftreten einer HTN-C beschrieben. Den eindeutig wichtigsten Risikofaktor stellt dabei die medikamentöse NonAdhärenz dar. Es existieren jedoch bislang lediglich sehr limitierte Daten aus indirekten Adhärenzmessungen zum Grad der Non-Adhärenz bei Patienten mit HTN-C. Alle bisherigen Studien zu diesem Thema weisen wesentliche Einschränkungen, wie retrospektives Design oder Verwendung von ausschließlich indirekten Methoden zur Adhärenzbestimmung (Pillenzählung, Fragebögen, klinische Einschätzung oder Auswertung von Rezepteinlösungen) auf.

Adhärenzdaten aus direkten Adhärenztestungen mittels chemisch-toxikologischer Verfahren in dieser Hochrisiko-Patientengruppe fehlen bis dato vollständig. Eine sorgfältige Bewertung der Medikamentenadhärenz ist von entscheidender Bedeutung für das diagnostische und therapeutische Prozedere bei HTN-C und bestimmt damit in hohem Maße die Qualität der weiteren Patientenversorgung. Zusätzlich können viele, zum Teil nicht verschreibungspflichtige, Begleitmedikamente und weitere Stoffe zu einer Erhöhung des Blutdruckes beitragen.

In der vorliegenden Arbeit sollen folgende Aspekte bei Patienten mit HTN-C, die in einer Notaufnahme aufgenommen wurden, mittels direkter toxikologischer Nachweismethode untersucht werden: (1a) Wie hoch ist der prozentuale Anteil an non-adhärenten Patienten? (1b) Gibt es Prädiktoren, die auf das Vorliegen einer Non-Adhärenz in diesem Kollektiv hindeuten?; (2a) Wie hoch ist der prozentuale Anteil an Patienten in dem untersuchten Kollektiv, die potenziell blutdrucksteigernde Medikamente bzw. Stoffe eingenommen haben? (2b) Welche blutdrucksteigernden Substanzklassen spielen in dem untersuchten Kollektiv eine Rolle?

Die hieraus gewonnenen Daten könnten einen Beitrag leisten, die Diagnostik und Therapie von Patienten mit HTN-C zu verbessern. 


\section{Patienten und Methoden}

\subsection{Votum der Ethikkommission}

Der Prüfplan (Antragsnummer 6/6/14) wurde durch die Ethikkommission der Universitätsmedizin Göttingen genehmigt.

\subsection{Aufnahme in die Studie}

Die Aufnahme in die Studie erfolgte bei Patienten, welche die Einschlusskriterien erfüllten und keine Ausschlusskriterien aufwiesen. Informationen über die Teilnahme erhielten die Patienten durch ein Informationsblatt $\mathrm{zu}$ dieser Studie. Fragen wurden im Aufklärungsgespräch durch den Prüfarzt oder einer von ihm benannten Person beantwortet. Die Einwilligung der Patienten erfolgte schriftlich mittels einer standardisierten Erklärung.

\subsection{Studiendesign}

Es handelt sich im Folgenden um eine nicht-randomisierte, monozentrische Querschnittsstudie.

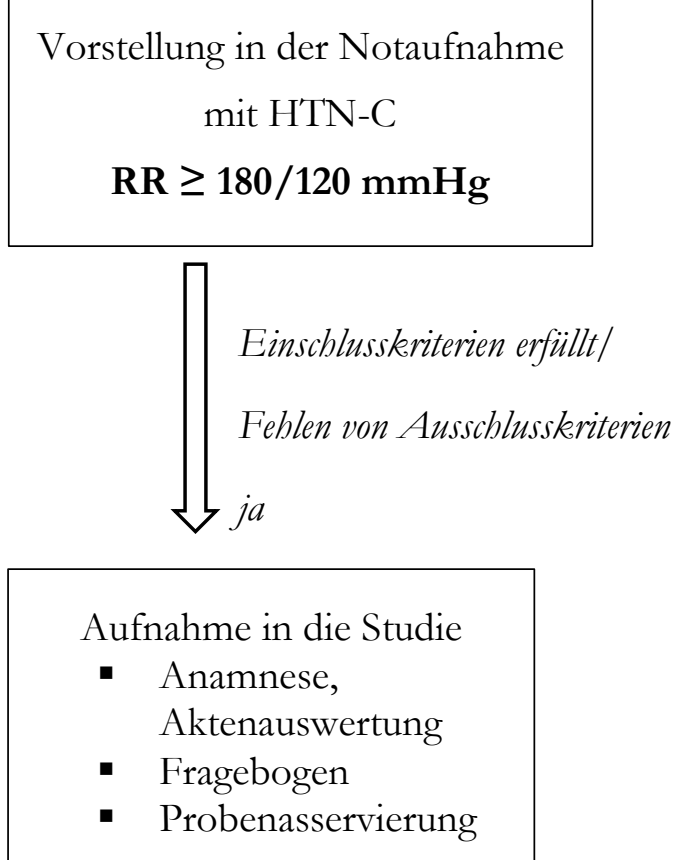

Abbildung 1: Flowchart der Studie. 


\subsection{Patienten}

Für die vorliegende Studie wurden 100 Patienten der interdisziplinären Notaufnahme der Universitätsmedizin Göttingen untersucht. Alle Patienten wurden mit HTN-C bzw. Notfall eingeliefert. Die Auswahl der Patienten erfolgte anhand der in Tabelle 2 definierten Kriterien.

Tabelle 2: Ein- und Ausschlusskriterien der Studie.

\begin{tabular}{|l|l|}
\hline \multicolumn{1}{|c|}{ Einschlusskriterien } & \multicolumn{1}{c|}{ Ausschlusskriterien } \\
\hline $\begin{array}{l}\text { Blutdruck } \geq 180 \mathrm{mmHg} \text { systolisch und/oder } \\
\geq 120 \mathrm{mmHg} \text { diastolisch }\end{array}$ & Alter $<18$ Jahre \\
\hline Einwilligungsfähigkeit & Ablehnung der Studie \\
\hline Unterschriebene Einverständniserklärung & $\begin{array}{l}\text { Primärer Aufnahmegrund Kopfverletzung } \\
\text { oder cerebrovaskulärer Notfall }\end{array}$ \\
\hline & Stammzell- oder Knochenmarktransplantation \\
\hline & Schwangerschaft \\
\hline
\end{tabular}

\subsection{Blutdruckmessung}

Die initiale Blutdruckmessung wurde vom Pflegepersonal in der Notaufnahme vor Verabreichung von blutdrucksenkenden Medikamenten durchgeführt. Der Blutdruck wurde standardisiert unter Verwendung des Propaq ${ }^{\circledR}$ CS-Monitors mit angepasster FlexiPort Einschlauchmanschette der Firma Welch Allyn (Skaneateles Falls, New York, USA) in Herzhöhe bei Patienten in halbliegender Position auf einer Notfallliege gemessen.

\subsection{Datenerhebung}

Die Datenerhebung erfolgte von Oktober 2014 bis Juni 2015 gemeinsam mit einer zweiten Doktorandin (Elena Lowin) aus der Abteilung für Psychosomatische Medizin und Psychotherapie der Universitätsmedizin Göttingen.

Die klinischen Daten der Patienten wurden gemeinschaftlich mit der oben genannten Doktorandin erhoben. 


\subsubsection{Gemeinsam erhobene Parameter}

Die soziodemographischen Daten (Geschlecht, Alter, Familienstand, Anzahl der Kinder, berufliche Tätigkeit) und aktuelle Anamnese der Patienten (systolischer und diastolischer Blutdruck, Symptome) einschließlich der ausführlichen Medikamentenanamnese (Kapitel 2.6.4) wurden gemeinsam erhoben und ausgewertet. Auch die folgenden potenziellen Risikofaktoren für das Auftreten einer HTN-C wurden gemeinsam bewertet: sekundäre Ursachen der HTN, Vorliegen von relevanten kardiovaskulären Risikofaktoren und Krankheiten, Body-Mass-Index (BMI), Alkohol- oder Nikotinkonsum und relevante Begleiterkrankungen.

\subsubsection{Nephrologische Parameter}

Nephrologische Parameter beinhalten die selbstberichtete Einnahme von potenziell störenden Nahrungsmitteln in Kombination mit blutdrucksenkenden Medikamenten (Pampelmuse, Knoblauch, Broccoli, andere Kohlsorten, Zimt, Bärlauch, Johanniskraut, Lakritze) bis 48 Stunden vor Eintritt in die Notaufnahme sowie die Bestimmung der Nierenfunktion mittels Abschätzung (e = estimated) der glomerulären Filtrationsrate (GFR) (Kapitel 2.6.5) und die Klassifikation der chronischen Nierenerkrankung mittels eGFR und Albuminurie.

Die toxikologische Urinanalyse (Kapitel 2.8) wurde zur direkten Bestimmung der Medikamentenadhärenz im Rahmen der hier vorliegenden Dissertation in der Klinik für Nephrologie und Rheumatologie durchgeführt.

\subsubsection{Psychosomatische Parameter}

Im Rahmen der Dissertationsarbeit von Elena Lowin erfolgte die indirekte Messung der Medikamentenadhärenz (Medication Adherence Report Scale, Rief Adherence Index) sowie die Erfassung der Befindlichkeit und Adhärenz-relevanter Faktoren mittels ausgewählter Fragebögen (Anhang).

\subsubsection{Medikamentenanamnese}

Am Einschlusstag erfolgte eine ausführliche Befragung der Patienten hinsichtlich ihrer Medikamenteneinnahme. Dabei wurden alle Medikamentenklassen berücksichtigt. Eine Darstellung der ausgewerteten Inhalte zur antihypertensiven Medikation zeigt Tabelle 3. 
Tabelle 3: Strukturierte Abfrage der antihypertensiven Medikation.

\begin{tabular}{|l|}
\hline Jahre seit Beginn der Bluthochdruck-Therapie \\
\hline Name der verschriebenen Substanz(en) \\
\hline Anzahl der blutdrucksenkenden Medikamente \\
\hline Verwendung von Kombinationspräparaten \\
\hline Einnahme nach Medikationsplan \\
\hline
\end{tabular}

Zusätzlich wurde der Zeitpunkt der Gabe von notfallmäßig in der Akutsituation gegebenen blutdrucksenkenden Medikamenten (mehr bzw. weniger als 30 min. vor Abgabe der Urinprobe) erfasst, um die Therapieadhärenz sicher beurteilen zu können. Neben der von behandelnden Ärzten verschriebenen Medikation wurde auch die passagere Einnahme weiterer Medikamente, insbesondere Schmerzmittel, oder pflanzlicher Präparate erfragt.

\subsubsection{Labordiagnostik}

Die Blutentnahme erfolgte im Rahmen der Routine durch das Personal der Notaufnahme. Die im Zentrallabor der Universitätsmedizin Göttingen ermittelten Werte (Natrium, Kalium, Kreatinin, Glucose, C-reaktives Protein [CRP] und Thyreoidea-stimulierendes Hormon [TSH]) wurden nachträglich der Akte entnommen. Die eGFR wurde mittels der MDRDFormel (entnommen aus der Modification-of-Diet-in-Renal-Disease-Studie) berechnet:

$$
\begin{gathered}
\text { eGFR }\left(\mathrm{ml} / \mathrm{min} / 1,73 \mathrm{~m}^{2}\right)=186 \times \text { Serumkreatinin }^{-1,154} \times \text { Alter }^{-0,203} \times \text { Korrekturfaktor* }^{*} \\
\quad * 0,742 \text { bei Frauen; } 1,21 \text { bei Menschen mit schwarzer Hautfarbe }
\end{gathered}
$$

\subsection{Probenasservierung}

Für die Studie wurden ca. $50 \mathrm{ml}$ Urin pro Patient benötigt. Die folgenden Proben wurden bei Aufnahme in die Studie entnommen: 8,5 ml Urin-Monovette ${ }^{\circledR}$ (Firma Sarstedt, Nümbrecht, Deutschland); 3-mal $15 \mathrm{ml}$ Röhre, 120×17 mm, PP (Firma Sarstedt, Nümbrecht, Deutschland). Die Urin-Monovette wurde unmittelbar nach Gewinnung in das Labor transportiert. Aus dem Spontanurin der Patienten erfolgte eine Analyse von Urinstatus, Sediment und Proteinausscheidung. Die Parameter wurden im Rahmen der Routinediagnostik im nephrologisch-rheumatologischen Labor der Universitätsmedizin Göttingen bestimmt. Die $15 \mathrm{ml}$ Röhrchen wurden bis zur chemisch-toxikologischen 
Untersuchung des Urins bei $-80^{\circ} \mathrm{C}$ gelagert. Die Kennzeichnung der Proben erfolgte durch Codierung mit Pseudonym.

\subsection{Toxikologische Urinanalyse}

Die Studie wurde durch eine systematische toxikologische Untersuchung des Urins mittels Gaschromatographie-Massenspektrometrie (GC-MS) unterstützt. Die technische Durchführung der Urinanalysen erfolgte dabei durch das Toxikologische Labor der Universitätsmedizin Göttingen (Leitender Chemotechniker Hartmud Neurath).

\subsubsection{Analysegeräte und Bedingungen}

Die Analyse wurde unter Verwendung eines GC-MS-Systems der Firma Agilent Technologies Deutschland (Waldbronn), bestehend aus einem Gaschromatograph 66890N mit Split/Splitless-Injektor und Autosampler (7683B) gekoppelt mit einem QuadrupolMassenspektrometer (MSD 5975C) durchgeführt.

GC-Parameter: Injektor: Splitless 0,75 min, $270{ }^{\circ} \mathrm{C}$; Trennsäule: OPTIMA® 5 MS Accent (Macherey-Nagel, Düren, Deutschland); Trägergas: He, $1 \mathrm{ml} / \mathrm{min}$, Konstant-Flow-Modus; Ofentemperaturprogramm: $100{ }^{\circ} \mathrm{C}(2 \mathrm{~min})-30{ }^{\circ} \mathrm{C} / \mathrm{min}-340{ }^{\circ} \mathrm{C}(3 \mathrm{~min})$.

MS-Parameter: Ionisation: Electron Impact (EI), Full-Scan-Modus: 44 - 550 u.

\subsubsection{Vorbereitung der Proben}

Die Urinproben wurden nativ alkalisch mit einem organischen Lösungsmittelgemisch extrahiert. Teile der Probe wurden einer Spaltung metabolischer Addukte durch salzsaure Hydrolyse unterworfen. Nach Extraktion und Derivatisierung mit Acetanhydrid wurden die Probenteile vereint und gaschromatographisch untersucht. Für den Nachweis hydrophiler Wirkstoffe, z. B. ACE-Hemmer und Diuretika, wurde ein dritter Probenteil vor der Gaschromatographie einer extraktiven Alkylierung unterzogen.

\subsubsection{Auswertung}

Die gewonnenen Extrakte wurden mittels GC-MS im Full-Scan-Modus analysiert. Die Identifizierung der Arzneistoffe erfolgte durch Massenspektrenvergleich mit verschiedenen Referenzbibliotheken (Maurer et al. 2017; NIST 2017; Rösner et al. 2017). 
Anhand einer nach der Datenerhebung erstellten Medikamentenliste wurde für jede verschriebene Substanz eines Patienten eine der in Tabelle 4 genannten Angaben als Ergebnis erhalten.

Tabelle 4: Ergebnis der toxikologischen Analyse.

\begin{tabular}{|c|c|c|}
\hline Angabe & Interpretation & Arzneistoffe \\
\hline $\begin{array}{l}\text { Nachgewiesen bzw. } \\
\text { nicht nachweisbar }\end{array}$ & $\begin{array}{c}\text { Das Medikament wurde im Urin } \\
\text { des Patienten nachgewiesen/ } \\
\text { der Patient wird für dieses } \\
\text { Medikament als adhärent bewertet } \\
\text { bzw. } \\
\text { Das Medikament wurde im Urin } \\
\text { des Patienten nicht } \\
\text { nachgewiesen/ } \\
\text { der Patient wird für dieses } \\
\text { Medikament als non-adhärent } \\
\text { bewertet }\end{array}$ & $\begin{array}{l}\text { Ramipril, Enalapril, Benazepril, } \\
\text { Valsartan, Irbesartan, Losartan, } \\
\text { Metoprolol, Bisoprolol, } \\
\text { Propranolol, Lercanidipin, } \\
\text { Amlodipin, Felodipin, } \\
\text { Nitrendipin, Verapamil, Urapidil, } \\
\text { Torasemid, Furosemid, Piretanid, } \\
\text { Hydrochlorothiazid, Xipamid, } \\
\text { Triamteren, Eplerenon, } \\
\text { Spironolacton }\end{array}$ \\
\hline $\begin{array}{c}\text { Nur in hoher } \\
\text { Konzentration erfasst }\end{array}$ & $\begin{array}{l}\text { Das Medikament wird nur in } \\
\text { hoher Konzentration im Urin } \\
\text { erfasst/ } \\
\text { die Adhärenz für dieses } \\
\text { Medikament ist nicht sicher } \\
\text { beurteilbar }\end{array}$ & $\begin{array}{c}\text { Nebivolol, Carvedilol, Aliskiren, } \\
\text { Moxonidin }\end{array}$ \\
\hline $\begin{array}{l}\text { Durch die Methode } \\
\text { nicht erfasst }\end{array}$ & $\begin{array}{l}\text { Das Medikament wird durch die } \\
\text { Methode nicht erfasst/ } \\
\text { die Adhärenz für dieses } \\
\text { Medikament ist nicht beurteilbar }\end{array}$ & $\begin{array}{l}\text { Lisinopril, Telmisartan, } \\
\text { Candesartan, Doxazosin, } \\
\text { Isosorbiddinitrat }\end{array}$ \\
\hline $\begin{array}{c}\text { Zusätzlich } \\
\text { nachgewiesen }\end{array}$ & $\begin{array}{c}\text { Das Medikament bzw. } \\
\text { Nahrungsmittel wurde zusätzlich } \\
\text { im Urin des Patienten } \\
\text { nachgewiesen/ } \\
\text { die Substanz ist möglicherweise } \\
\text { an einer Verschlechterung der } \\
\text { Blutdruckwerte beteiligt }\end{array}$ & \\
\hline
\end{tabular}


Einschränkung: Die Adhärenz für im Urin nachgewiesene Medikamente, die mehr als 30 min. vor Abgabe der Urinprobe durch den (Not-)Arzt verabreicht wurden ist nicht sicher beurteilbar.

\subsection{Statistik}

Die statistische Auswertung wurde vom Institut für Medizinische Statistik der Universitätsmedizin Göttingen begleitet. Die Analyse erfolgte mit Hilfe des Statistikprogramms Statistica 12 und Microsoft Excel 2013. Patientendaten wurden zu einem Zeitpunkt an nur einem Kollektiv erhoben. Über eine graphische Darstellung wurde die Normalverteilung geprüft. Als deskriptive Mittel dienten abhängig vom Skalenniveau sowie dem Vorliegen bzw. der Abwesenheit einer Normalverteilung der Variablen entweder Anzahl und Prozente (z. B. Geschlecht) oder Mittelwerte und Standardabweichung (SD) bzw. Mediane mit Interquartilsabstand (IQR) (z. B. Alter). Interessierende Variablen wurden nach Bewertung der Therapieadhärenz zwischen den Gruppen „Adhärent“ und „Nonadhärent“ verglichen und auf statistische Unterschiede überprüft. Zur Beurteilung der Signifikanz kam für metrische Variablen der t-Test, für dichotome Variablen der Exakte Fisher-Test (zweiseitig) und für kategoriale Variablen Pearson's Chi-Quadrat-Test zum Einsatz. Balken- und Säulendiagramme wurden zur graphischen Veranschaulichung berechneter Werte angefertigt. Der Zusammenhang zwischen Glucose und Blutdruck wurde durch Spearmans Rangkorrelation quantifiziert. Eine logistische Regressionsanalyse sollte die Wahrscheinlichkeit für Non-Adhärenz schätzen. Dabei galt als abhängige Variable die Gruppenzugehörigkeit „Adhärent“ oder „Non-adhärent“. Merkmale mit möglichem Einfluss auf die Medikamenteneinnahme wurden als unabhängige Variable eingesetzt. Für alle statistischen Tests wurde ein p-Wert $<0,05$ als signifikantes Ergebnis betrachtet.

\subsection{Datenschutz}

Die Datenerhebung, -speicherung und -auswertung erfolgte pseudonymisiert. Die Codierung der Proben erfolgte durch fortlaufende Nummernvergabe. Alle ermittelten Daten unterliegen dem Datenschutz. Personenbezogene Daten (außer Geschlecht und Alter) werden nicht weitergegeben. 


\section{Ergebnisse}

\subsection{Patienten}

Während des Untersuchungszeitraumes wurden insgesamt 100 Patienten mit HTN-C bzw. Notfall, die in der interdisziplinären Notaufnahme der Universitätsmedizin Göttingen aufgenommen wurden, in die vorliegende Studie eingeschlossen.

\subsubsection{Soziodemographische Daten}

Das Patientenkollektiv setzte sich aus 54 Frauen (54\%) und 46 Männern (46\%) im Alter von 23 bis 90 Jahren zusammen. Das Durchschnittsalter der Patienten lag bei 66,5 \pm 12,9 Jahren. Tabelle 5 gibt einen Überblick über die sozialen Merkmale des Kollektivs.

Tabelle 5: Soziodemographische Merkmale.

\begin{tabular}{|l|c|}
\hline Patienten (n) & 100 \\
\hline Geschlecht, n (\%) & $54(54 \%)$ \\
weiblich & $46(46 \%)$ \\
männlich & $66,5 \pm 12,9$ \\
\hline Alter (Jahre), Mittelwert \pm SD & $10(10 \%)$ \\
\hline Familienstand, n (\%) & $3(3 \%)$ \\
ledig & $60(60 \%)$ \\
feste Partnerschaft & $9(9 \%)$ \\
verheiratet & $17(17 \%)$ \\
getrennt/geschieden & $1(1 \%)$ \\
verwitwet & $2(1-2)$ \\
keine Angabe & \\
\hline Anzahl der Kinder, Median (IQR) & \\
\hline
\end{tabular}




\begin{tabular}{|l|c|}
\hline Patienten (n) & 100 \\
\hline Berufliche Tätigkeit, n (\%) & \\
selbstständig & $4(4 \%)$ \\
angestellt & $20(20 \%)$ \\
arbeitslos & $9(9 \%)$ \\
Rentner(in)/Ruhestand & $64(64 \%)$ \\
keine Angabe & $3(3 \%)$ \\
\hline
\end{tabular}

\subsubsection{Klinische Daten}

Als Kriterium für die Aufnahme in die Studie wurde ein initial erhöhter Blutdruck $\geq 180 \mathrm{mmHg}$ systolisch und/oder $\geq 120 \mathrm{mmHg}$ diastolisch definiert. Im Mittel wurden systolische Werte von $201 \pm 18 \mathrm{mmHg}$ und diastolische Werte von $106 \pm 18 \mathrm{mmHg}$ gemessen (Tabelle 6). Der höchste gemessene systolische Blutdruck betrug $250 \mathrm{mmHg}$, der höchste diastolische Blutdruck lag bei $150 \mathrm{mmHg}$.

Tabelle 6: Initiale Blutdruckwerte.

\begin{tabular}{|l|c|}
\hline Patienten (n) & 100 \\
& Mittelwert \pm SD \\
\hline Systolischer Blutdruck (mmHg) & $201 \pm 18$ \\
Diastolischer Blutdruck (mmHg) & $106 \pm 18$ \\
\hline
\end{tabular}

Bei 13 der eingeschlossenen Patienten (13\%) zeigten sich hypertensive Endorganschäden, im Sinne eines hypertensiven Notfalls. Die entsprechenden Endorganschäden sind mit Angabe ihrer beobachteten Häufigkeit in Tabelle 7 aufgeführt. 
Tabelle 7: Manifestationen von Endorganschäden und deren Häufigkeit bei hypertensiven Notfällen.

\begin{tabular}{|l|c|}
\hline Patienten (n) & $\mathbf{1 0 0}$ \\
& $\mathrm{n}(\%)$ \\
\hline Lungenödem & $1(1 \%)$ \\
\hline Akutes Koronarsyndrom & $9(9 \%)$ \\
\hline Neurologische Veränderungen & $3(3 \%)$ \\
\hline
\end{tabular}

Die Symptomatik bei Vorstellung in der Notaufnahme wurde bei allen Patienten offen erfragt. Das meistgenannte Symptom war mit 33\% Brustschmerzen. Fast genauso häufig klagten die Patienten über Schwindel (30\%). Dyspnoe wurde von 21\% der Patienten als Symptom beschrieben, Übelkeit/Erbrechen von 20\% der Patienten. Die weitere Verteilung der kategorisierten Antworten ist in Abbildung 2 dargestellt. 


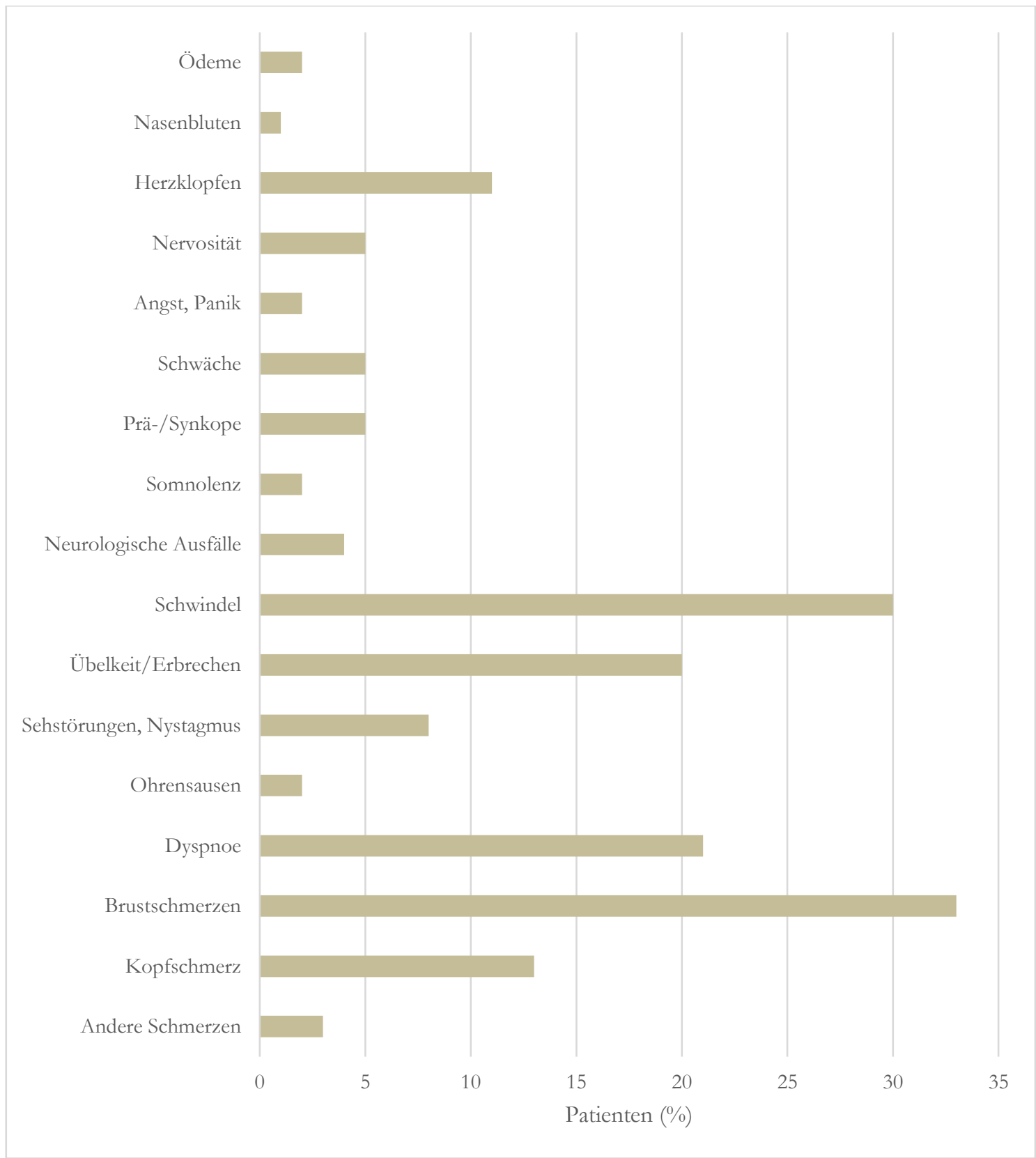

Abbildung 2: Symptome bei Vorstellung in der Notaufnahme, $\mathrm{n}=100$.

Bei 90 Patienten (90\%) lag eine bekannte primäre HTN vor, bei 4 Patienten (4\%) eine sekundäre Form der HTN. Die Erstdiagnose wurde im Median vor 11 Jahren (IQR 3-20) gestellt. Von den insgesamt 94 Patienten mit vorbekannter HTN gaben 29 (30,8\%) an auch im Alltag unter Beschwerden durch den erhöhten Blutdruck zu leiden. Bei 6 Patienten (6\%) wurden erstmalig zu hohe Blutdruckwerte gemessen. Zusätzlich zur HTN fanden sich bei 68 Patienten (68\%) anamnestisch weitere kardiovaskuläre Risikofaktoren oder Krankheiten. Der durchschnittliche BMI lag mit 29,1 $\pm 6,9 \mathrm{~kg} / \mathrm{m}^{2}$ im prä-adipösen Bereich, 37 Patienten $(37 \%)$ waren definitionsgemäß (BMI $\left.\geq 30 \mathrm{~kg} / \mathrm{m}^{2}\right)$ adipös. Eine Anzahl von 21 Patienten 
(21\%) waren aktiv und 40 Patienten (40\%) waren ehemalige Raucher. Relevante anamnestische Daten sind in Tabelle 8 zusammengefasst.

Tabelle 8: Patientencharakteristika.

\begin{tabular}{|l|c|}
\hline Patienten (n) & 100 \\
\hline Kardiovaskuläre Risikofaktoren und Krankheiten, n (\%) & $27(27 \%)$ \\
Koronare Herzkrankheit & $6(6 \%)$ \\
Herzinsuffizienz & $5(5 \%)$ \\
Periphere arterielle Verschlusskrankheit & $10(10 \%)$ \\
Chronische Niereninsuffizienz & $14(14 \%)$ \\
Schlaganfall & $22(22 \%)$ \\
Diabetes mellitus & $29(29 \%)$ \\
Hyperlipidämie & $29,1 \pm 6,9$ \\
\hline BMI (kg/m ${ }^{2}$, Mittelwert \pm SD & $37(37 \%)$ \\
\hline BMI $\geq 30$ kg/m ${ }^{2}, \mathrm{n}(\%)$ & $44(44 \%)$ \\
\hline Körperliche Aktivität, n (\%) & $21(21 \%)$ \\
\hline >2 Stunden/Woche & $20(20 \%)$ \\
\hline Regelmäßiger Alkoholkonsum, n (\%) & $61(61 \%)$ \\
\hline Raucheranamnese, n (\%) & \\
\hline
\end{tabular}

Die medizinische Behandlung der HTN-C konnte bei 54 Patienten (54\%) ambulant erfolgen. Bei 46 Patienten (46\%) war eine stationäre Weiterbehandlung notwendig: 28 Patienten (28\%) wurden von der interdisziplinären Notaufnahme auf eine Normalstation verlegt, 11 Patienten (11\%) auf eine Chest Pain Unit und 7 Patienten (7\%) auf eine Intermediate Care/Intensive Care Unit.

\subsubsection{Antihypertensive Medikation}

Von den insgesamt 100 Patienten erhielten 86 (86\%) eine vom Hausarzt verschriebene antihypertensive Medikation. Die blutdrucksenkende Therapie wurde im Median vor 10 Jahren (IQR 2-20) begonnen. Im Durchschnitt sollten Patienten 2,15 \pm 1,45 
Antihypertensiva einnehmen. Bei 28 von 86 Patienten (32,6\%) wurden

Kombinationspräparate verwendet. Die verschriebenen Substanzklassen der Antihypertensiva zeigt Tabelle 9.

Tabelle 9: Antihypertensive Substanzklassen.

\begin{tabular}{|l|c|}
\hline Patienten (n) & $\mathbf{8 6}$ \\
\hline ACE-Hemmer & $39(45,4 \%)$ \\
\hline Angiotensin-II-Blocker & $38(44,2 \%)$ \\
\hline Betablocker & $56(65,1 \%)$ \\
\hline Renininhibitoren & $2(2,3 \%)$ \\
\hline Kalziumantagonisten & $32(37,2 \%)$ \\
\hline Alpha-2-Rezeptoragonisten & $11(12,8 \%)$ \\
\hline Alphablocker & $5(5,8 \%)$ \\
\hline Nitrate & $2(2,3 \%)$ \\
\hline Schleifendiuretika & $13(15,1 \%)$ \\
\hline Thiazide & $36(41,9 \%)$ \\
\hline Kaliumsparende Diuretika & $2(2,3 \%)$ \\
\hline Aldosteronantagonisten & $3(3,5 \%)$ \\
\hline
\end{tabular}

\subsubsection{Laborparameter}

Die Laborparameter Natrium, Kalium, Glucose, CRP und TSH zum Zeitpunkt der Aufnahme in der Notaufnahme sind zur Übersicht in Tabelle 10 dargestellt. Der GlucoseWert im Blut wurde nicht nüchtern bestimmt. 
Tabelle 10: Laborparameter.

\begin{tabular}{|l|c|}
\hline Patienten (n) & 100 \\
\hline Natrium (mmol/l) & Mittelwert \pm SD \\
\hline Kalium (mmol/l) & $138 \pm 5$ \\
\hline Glucose (mg/dl) & $3,9 \pm 0,5$ \\
\hline CRP (mg/l) & $126 \pm 35$ \\
\hline TSH (mlU/l) & $10,1 \pm 19,4$ \\
\hline
\end{tabular}

\subsection{Nierenfunktion}

Anamnestisch konnte bei 10 Patienten (10\%) eine chronische Niereninsuffizienz erhoben werden. Die Serumkreatininwerte zum Zeitpunkt der Aufnahme lagen für die Frauen des Kollektivs bei $0,89 \pm 0,58 \mathrm{mg} / \mathrm{dl}(\mathrm{n}=54)$ und für die Männer bei $1,02 \pm 0,44 \mathrm{mg} / \mathrm{dl}$ ( $n=45)$. Zur Evaluation der Nierenfunktion wurde die eGFR der Patienten mittels der MDRD-Formel bestimmt.

Tabelle 11: Geschätzte glomeruläre Filtrationsrate.

\begin{tabular}{|c|c|}
\hline $\begin{array}{l}\text { Patienten (n) } \\
\text { eGFR }\left(\mathrm{ml} / \mathrm{min} / 1,73 \mathrm{~m}^{2}\right)\end{array}$ & $\mathbf{9 9}$ \\
\hline$\geq 90$ & $\mathrm{n}(\%)$ \\
\hline $60-89$ & $43(43,4 \%)$ \\
\hline $30-59$ & $39(39,4 \%)$ \\
\hline $15-29$ & $14(14,1 \%)$ \\
\hline$<15$ & $2(2,0 \%)$ \\
\hline
\end{tabular}

Die Auswertung der Albuminausscheidung im Urin ( $\mathrm{n}=97)$ zeigte bei 40 Patienten $(41,2 \%)$ eine Mikroalbuminurie (30-300 mg/g Kreatinin) und bei 13 Patienten (13,4\%) eine Makroalbuminurie (> $300 \mathrm{mg} / \mathrm{g}$ Kreatinin). Bei 41 der insgesamt 43 Patienten mit normaler 
exkretorischer Nierenfunktion (eGFR $\geq 90 \mathrm{ml} / \mathrm{min} / 1,73 \mathrm{~m}^{2}$ ) (Tabelle 11) konnten zusätzlich Daten zur Proteinurie erhoben werden. Es fanden sich unter den Patienten mit normaler exkretorischer Nierenfunktion 19 Patienten (46,3\%) mit Mikro- und 2 Patienten $(4,9 \%)$ mit Makroalbuminurie. Ebenso konnten bei 38 der insgesamt 39 Patienten mit leicht eingeschränkter exkretorischer Nierenfunktion (eGFR $60-89 \mathrm{ml} / \mathrm{min} / 1,73 \mathrm{~m}^{2}$ ) Daten zur Proteinurie erhoben werden. Es fanden sich unter den Patienten mit leicht eingeschränkter exkretorischer Nierenfunktion 13 Patienten (34,2\%) mit Mikro- und 5 Patienten (13,2\%) mit Makroalbuminurie. Vor diesem Hintergrund lassen sich 56 von 99 Patienten (56,6\%) einem Stadium G1-G5 der chronischen Nierenerkrankung zuordnen (3,0\% Fehlende Werte) (Abbildung 3).

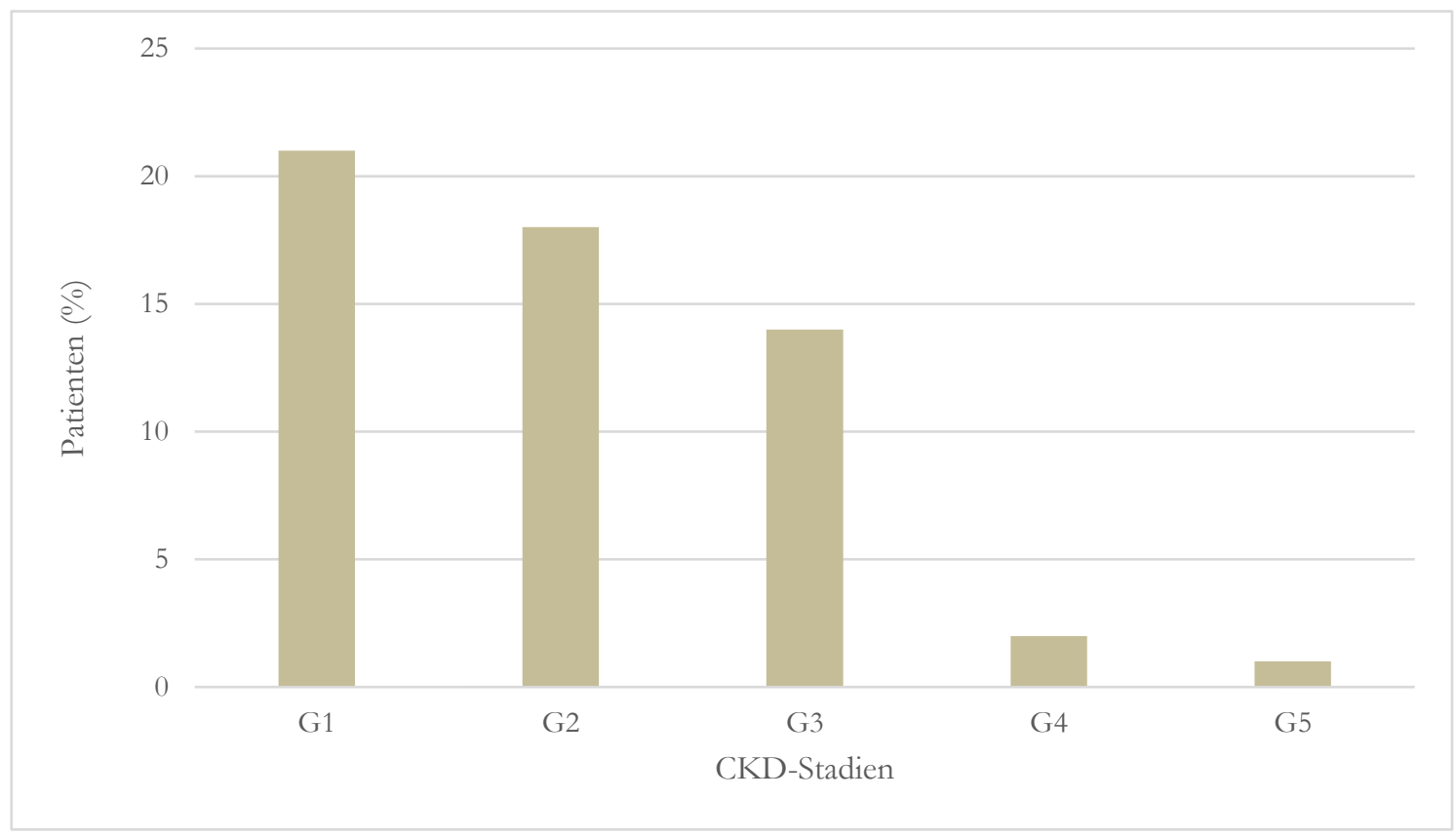

Abbildung 3: Verteilung der Patienten auf die Stadien G1-G5 der chronischen Nierenerkrankung (chronic kidney disease; $\mathrm{CKD}), \mathrm{n}=99$.

\subsection{Beurteilung der Therapieadhärenz}

Die Therapieadhärenz der 86 Patienten mit antihypertensiver Vormedikation wurde mittels toxikologischer Urinanalyse untersucht. In 24 Fällen verabreichte entweder der (Not-)Arzt blutdrucksenkende Medikamente in einem Zeitfenster, sodass keine valide Zuordnung der Daten der Urinanalyse zum Einnahmeverhalten der Patienten $(n=9)$ möglich war oder aufgrund von methodischen Einschränkungen der direkten toxikologischen Bestimmung einzelner antihypertensiver Substanzen (vgl. Kapitel 2.8.3) $(\mathrm{n}=14)$ konnte die Adhärenz nicht sicher beurteilt werden. Darüber hinaus konnte ein Patient den antihypertensiven 
Wirkstoff nicht nennen. Diese Patienten wurden von der Untersuchung ausgeschlossen.

Demnach konnten die Urinuntersuchungsdaten von 62 Patienten für die Adhärenzbewertung verwendet werden (Abbildung 4).

$$
\begin{aligned}
& 100 \text { Patienten mit HTN-C } \\
& \mathrm{RR} \geq 180 / 120 \mathrm{mmHg}
\end{aligned}
$$

14 Patienten ohne antihypertensive Vormedikation

86 Patienten: Toxikologische Untersuchung des Urins

auf verschriebene Antihypertensiva

24 Patienten: Adhärenz nicht sicher beurteilbar

62 Patienten: Bewertung der Therapieadhärenz

\begin{tabular}{|c|}
\hline 26 Patienten \\
$\mathbf{4 1 , 9 \%}$ \\
Adhärent \\
\hline
\end{tabular}

$$
\begin{array}{|c|}
\hline 36 \text { Patienten } \\
\mathbf{5 8 , 1 \%} \\
\text { Non-adhärent } \\
\hline
\end{array}
$$

Abbildung 4: Therapieadhärenz.

Von diesen 62 Patienten waren 26 (41,9\%) vollständig adhärent (d. h. alle verschriebenen blutdrucksenkenden Medikamente wurden im Urin gefunden) und 36 (58,1\%) non-adhärent: 21 Patienten $(33,9 \%)$ waren teilweise non-adhärent (d. h. mindestens ein verschriebenes blutdrucksenkendes Medikament wurde im Urin nicht gefunden), 15 Patienten (24,2\%) waren vollständig non-adhärent (keines der verschriebenen blutdrucksenkenden Medikamente wurde nachgewiesen). Der Grad der Adhärenz ist in Abbildung 5 gezeigt. 


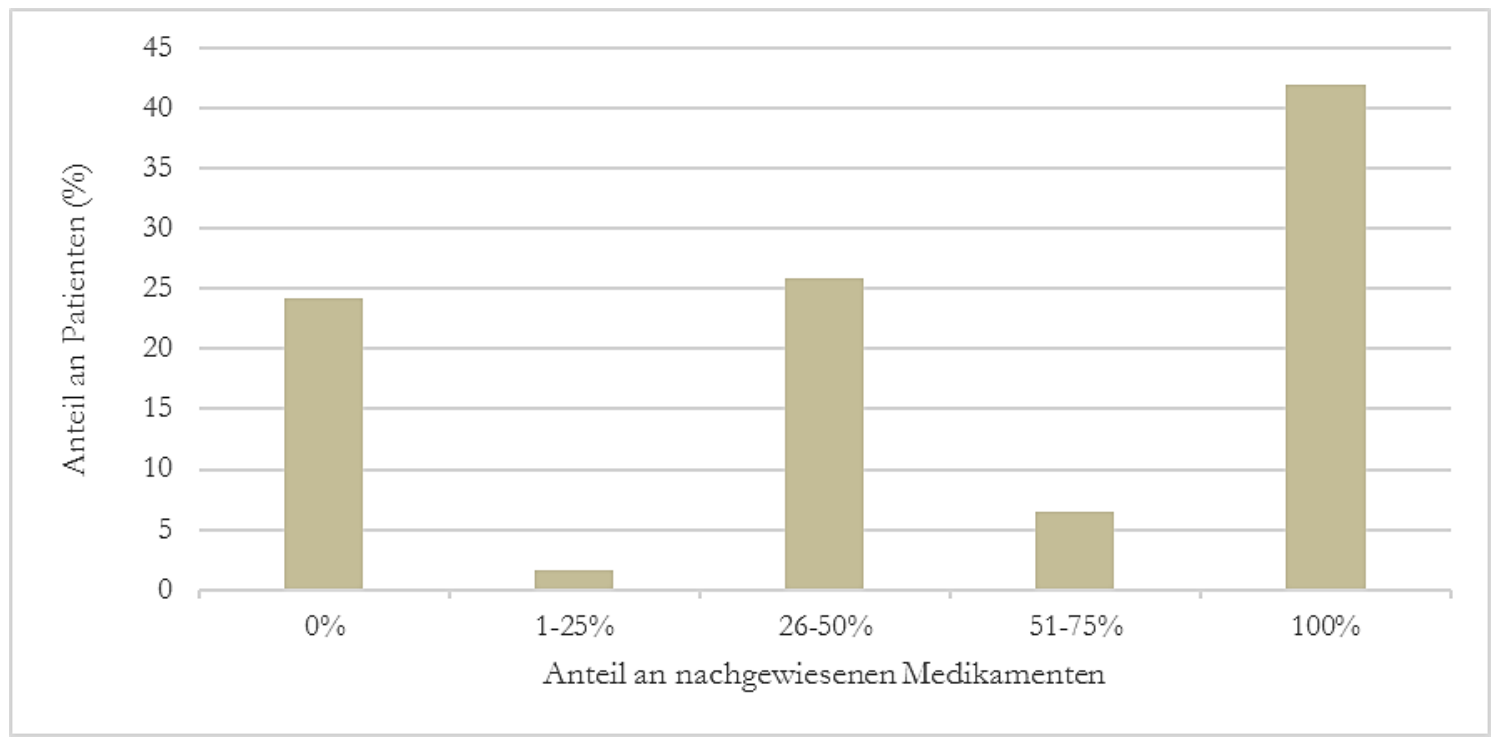

Abbildung 5: Verteilung des Adhärenzgrades, $\mathrm{n}=62$.

Es gab eine hohe Diskrepanz zwischen indirekter und direkter Adhärenzmessung in der vorliegenden Studie. Eine Anzahl von 75 von 86 Patienten (87,2\%) gaben vollständige Adhärenz innerhalb des Interviews an, während die Urinanalyse ergab, dass 26 von 62 Patienten $(41,9 \%)$ adhärent waren $(\mathrm{p}<0,01)$.

\subsubsection{Adhärenz in Bezug auf die Substanzklasse}

Im Vergleich der Substanzklassen variierte die Adhärenz von 50\% (Alphablocker) bis 84,6\% (Betablocker) (Abbildung 6). Während Betablocker gleichzeitig die am häufigsten verschriebenen Medikamente waren, zeigten Diuretika als zweithäufigste Substanzklasse und ACE-Hemmer als dritthäufigste Substanzklasse jeweils eine Adhärenz von 56,2\%. 


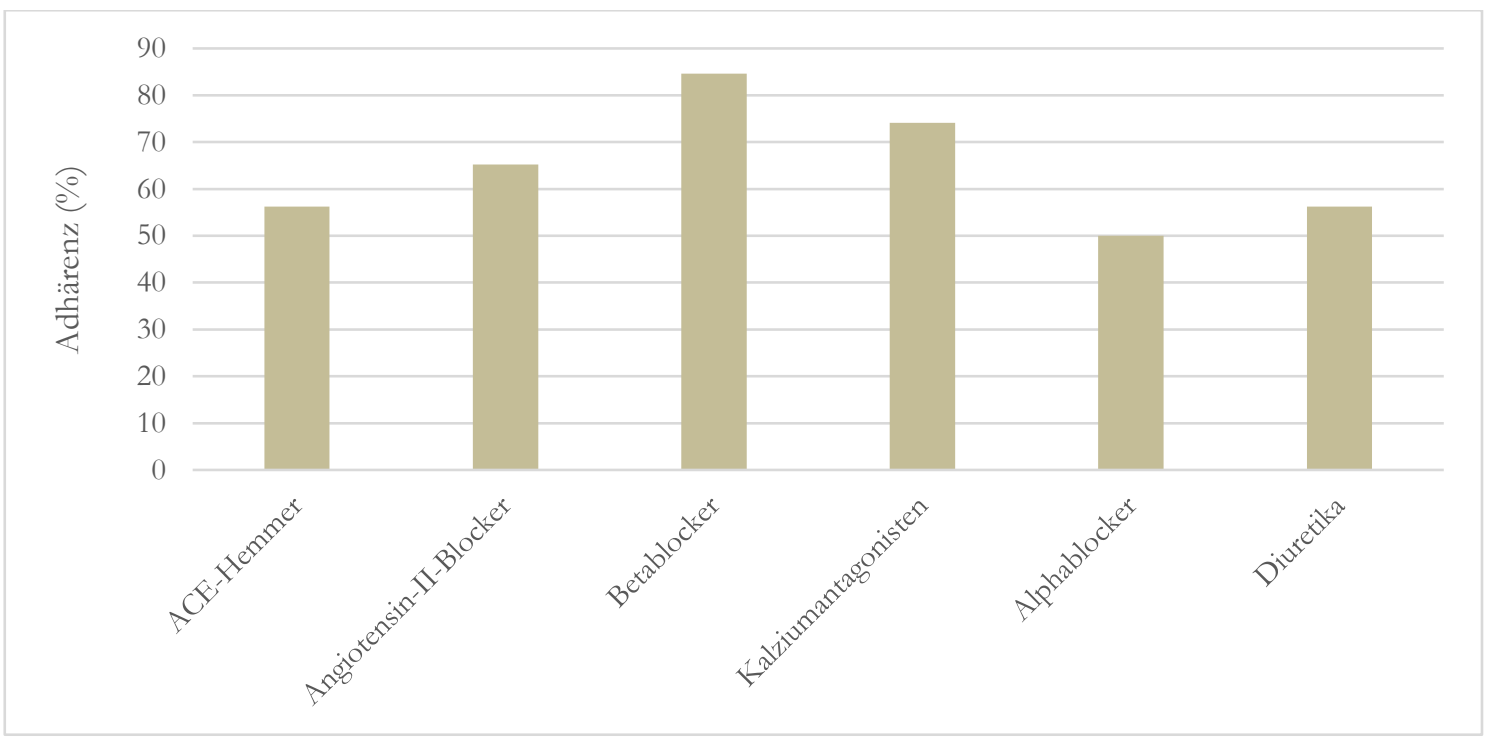

Abbildung 6: Therapieadhärenz in den verschiedenen Substanzklassen.

\subsection{Vergleich zwischen adhärenten und non-adhärenten Patienten}

Non-adhärente Patienten hatten eine signifikant längere HTN-Vorgeschichte (15 vs. 10 Jahre; $\mathrm{p}=0,04)$, bekamen mehr blutdrucksenkende Medikamente verschrieben (Anzahl 2,75 $\pm 1,05$ vs. $1,5 \pm 0,65 ; \mathrm{p}<0,01)$ und wiesen eine höhere Gesamtanzahl an Medikamenten auf $(7,11 \pm 3,37$ vs. $3,42 \pm 1,84 ; \mathrm{p}<0,01)$. Sie unterschieden sich von adhärenten Patienten hinsichtlich der Einnahme von Antidepressiva (6 vs. 0; $\mathrm{p}=0,04)$ und der Prävalenz mehrerer Komorbiditäten (Diabetes mellitus [11 vs. 0; $\mathrm{p}<0,01$ ], Herzinsuffizienz [6 vs. 0; $\mathrm{p}=0,04$ ], chronische Niereninsuffizienz anamnestisch [6 Patienten vs. 0 Patienten; $p=0,04]$ ). Es gab keine Unterschiede in Bezug auf Geschlecht, Alter, Blutdruck und Schweregrad der HTN-C, Behandlung mit einer Kombinationstherapie, koronare Herzkrankheit, periphere arterielle Verschlusskrankheit, früheren Schlaganfall, Hyperlipidämie, BMI oder Rauchverhalten. Beachtet man bei der Einordnung zur chronischen Nierenerkrankung die laborchemisch während der HTN-C erhobenen Daten der exkretorischen Nierenfunktion, gab es auch hier keinen signifikanten Unterschied. Die Daten sind in Tabelle 12 zusammengefasst. 
Tabelle 12: Vergleich zwischen adhärenten und non-adhärenten Patienten.

\begin{tabular}{|c|c|c|c|}
\hline & $\begin{array}{l}\text { Adhärent } \\
\qquad \mathrm{n}=26\end{array}$ & $\begin{array}{c}\text { Non-adhärent } \\
n=36\end{array}$ & $\mathrm{p}$ \\
\hline Geschlecht, n (\%) & & & 1,0 \\
\hline weiblich & $16(61,5 \%)$ & $23(63,9 \%)$ & \\
\hline männlich & $10(38,5 \%)$ & $13(36,1 \%)$ & \\
\hline Alter (Jahre) & $66,5 \pm 11,1$ & $68,4 \pm 12,6$ & 0,54 \\
\hline RR systolisch (mmHg) & $196 \pm 19$ & $200 \pm 14$ & 0,41 \\
\hline RR diastolisch (mmHg) & $109 \pm 21$ & $104 \pm 18$ & 0,28 \\
\hline Hypertensiver Notfall, n (\%) & $3(11,5 \%)$ & $4(11,1 \%)$ & 1,0 \\
\hline HTN-Vorgeschichte (Jahre) & $10(3-15)$ & $15(5-22)$ & 0,04 \\
\hline Anzahl Antihypertensiva & $1,5 \pm 0,65$ & $2,75 \pm 1,05$ & $<0,01$ \\
\hline Kombinationspräparate, $\mathrm{n}(\%)$ & $10(38,5 \%)$ & $10(27,8 \%)$ & 0,42 \\
\hline Antidepressive Begleittherapie, n (\%) & 0 & $6(16,7 \%)$ & 0,04 \\
\hline Gesamtanzahl Medikamente & $3,42 \pm 1,84$ & $7,11 \pm 3,37$ & $<0,01$ \\
\hline KHK, n (\%) & $4(15,4 \%)$ & $11(30,6 \%)$ & 0,23 \\
\hline Herzinsuffizienz, $\mathrm{n}(\%)$ & 0 & $6(16,7 \%)$ & 0,04 \\
\hline $\mathrm{pAVK}, \mathrm{n}(\%)$ & $1(3,8 \%)$ & $1(2,8 \%)$ & 1,0 \\
\hline \multicolumn{4}{|l|}{ Chronische Niereninsuffizienz, n (\%) } \\
\hline anamnestisch & 0 & $6(16,7 \%)$ & 0,04 \\
\hline evaluiert & $12(46,2 \%)$ & $23(63,9 \%)$ & 0,20 \\
\hline Schlaganfall, n (\%) & $5(19,2 \%)$ & $5(13,9 \%)$ & 0,72 \\
\hline Diabetes mellitus, n (\%) & 0 & $11(30,6 \%)$ & $<0,01$ \\
\hline Hyperlipidämie, n (\%) & $5(19,2 \%)$ & $12(33,3 \%)$ & 0,26 \\
\hline $\mathrm{BMI}\left(\mathrm{kg} / \mathrm{m}^{2}\right)$ & $28,3 \pm 7,5$ & $29,9 \pm 7,7$ & 0,44 \\
\hline Aktivraucher, n (\%) & $7(26,9 \%)$ & $4(11,1 \%)$ & 0,18 \\
\hline
\end{tabular}

Angaben als n (\%), Mittelwert \pm SD oder Median (IQR).

$\mathrm{KHK}=$ koronare Herzkrankheit, pAVK = periphere arterielle Verschlusskrankheit. 
Im Vergleich zwischen adhärenten und non-adhärenten Patienten fiel ein hoch signifikanter Gruppenunterschied hinsichtlich des Vorliegens eines Diabetes mellitus auf. In der Korrelationsanalyse zwischen Höhe des Blutdruckes zum Zeitpunkt der HTN-C und den gemessenen Blutglucosewerten konnte an dem vorliegenden Kollektiv kein Zusammenhang nachgewiesen werden (Tabelle 13).

Tabelle 13: Spearmans Rangkorrelation zwischen Glucose und Blutdruck; Korrelationskoeffizient rho $=\mathrm{r}$.

\begin{tabular}{|l|c|}
\hline & Glucose $(\mathrm{mg} / \mathrm{dl})$ \\
\hline RR systolisch $(\mathrm{mmHg})$ & $\mathrm{r}=0,07$ \\
\hline RR diastolisch $(\mathrm{mmHg})$ & $\mathrm{r}=-0,15$ \\
\hline
\end{tabular}

\subsection{Vorhersage für Non-Adhärenz}

Um die Eintrittswahrscheinlichkeit für Non-Adhärenz vorherzusagen, wurde im Rahmen der statistischen Auswertung eine (binäre) logistische Regression durchgeführt. Dabei wurden die folgenden unabhängigen Variablen in das Regressionsmodell aufgenommen: Geschlecht, Alter, Dauer der HTN-Behandlung, Blutdrucksenkende Medikamente pro Tag, Kombinationspräparate, Antidepressive Begleittherapie und Kardiovaskuläre Risikofaktoren und Krankheiten. Die Überprüfung des Modells insgesamt zeigte, dass sechs unabhängige Variablen anhand der vorliegenden Daten keinen Erklärungsbeitrag leisten. Diese Variablen wurden schrittweise aus dem Modell eliminiert. Nur die Variable Blutdrucksenkende Medikamente pro Tag hatte eine signifikante Einflussstärke: Steigt die Anzahl der blutdrucksenkenden Medikamente pro Tag um eine Einheit, so nimmt die relative Wahrscheinlichkeit für Non-Adhärenz um 19,7\% zu.

\subsection{Analyse potenzieller Wechselwirkungen}

\subsubsection{Nahrungsbestandteile}

Am Aufnahmetag wurden die Patienten nach der Einnahme verschiedener Nahrungsmittel bezogen auf die letzten zwei Tage befragt. Das Ergebnis ist in Abbildung 7 dargestellt. Zu den häufigsten Bestandteilen zählten Kohlsorten ( $\mathrm{n}=20)$, Knoblauch ( $\mathrm{n}=11$ ) und Lakritze $(\mathrm{n}=10)$. 


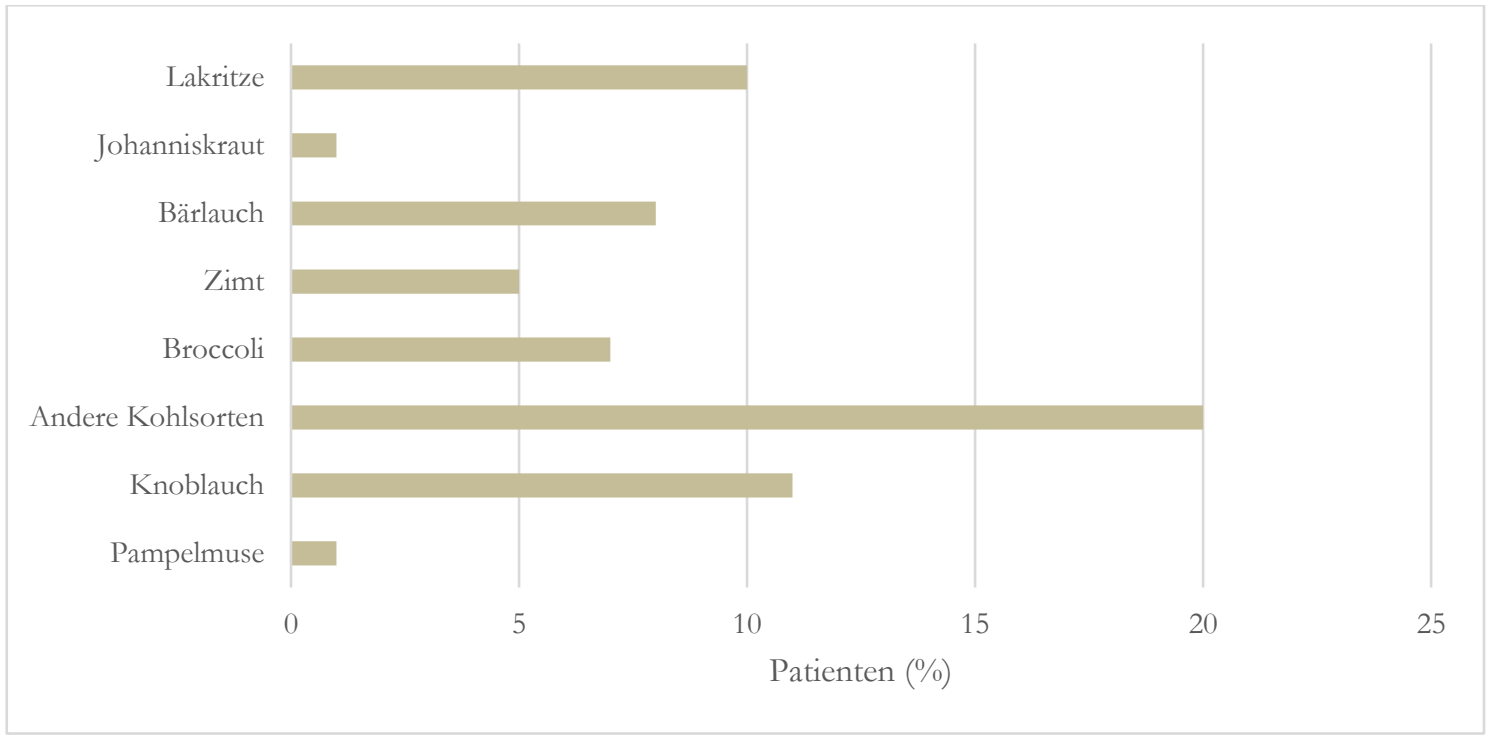

Abbildung 7: Einnahme bestimmter Nahrungsmittel, $\mathrm{n}=100$.

\subsubsection{Begleitmedikamente}

Neben der antihypertensiven Medikation wurden 8 Patienten Glucocorticoide verschrieben, 9 Patienten NSAIDs (Ibuprofen: $\mathrm{n}=6$; Diclofenac: $\mathrm{n}=2$; Naproxen: $\mathrm{n}=1$ ) und 10 Patienten Antidepressiva. Die Substanzklassen der verschriebenen Antidepressiva veranschaulicht Abbildung 8 .

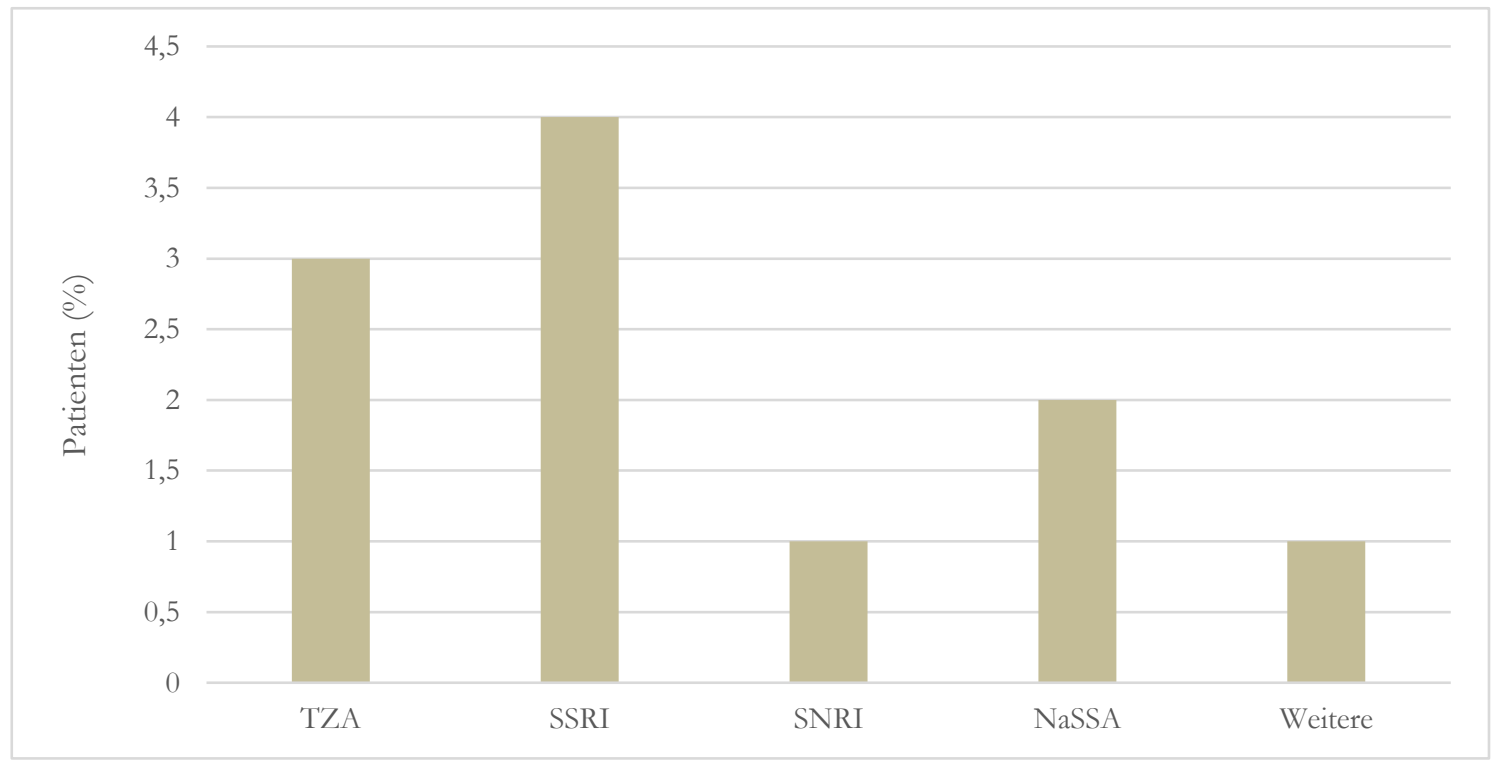

TZA = Trizyklische Antidepressiva, SSRI = Selektive Serotonin-Reuptake-Inhibitoren, SNRI = Selektive Serotonin-Noradrenalin-Reuptake-Inhibitoren, NaSSA = Noradrenerge und spezifisch serotonerge Antidepressiva. Weitere: Opipramol

Abbildung 8: Einnahme verschriebener Antidepressiva, $\mathrm{n}=100$. 


\subsubsection{Weitere nachgewiesene Substanzen}

Bei 96 von 100 Patienten konnten weitere, nicht angegebene, Arzneimittel bzw. Nahrungsoder Genussmittel nachgewiesen werden. Als potenziell blutdrucksteigernde Mittel wurden Coffein $(\mathrm{n}=92)$, Nikotin $(\mathrm{n}=20)$ und NSAIDs (Ibuprofen: $\mathrm{n}=14$; Diclofenac: $\mathrm{n}=6$; Ketoprofen: $\mathrm{n}=1$; Naproxen: $\mathrm{n}=1$; Indometacin: $\mathrm{n}=1$; Etoricoxib: $\mathrm{n}=1$ ) nachgewiesen. Darüber hinaus konnte bei fünf Patienten die Einnahme von niedrig dosierter Acetylsalicylsäure (ASS) nachgewiesen werden. Außerdem wurden Arzneimittel mit ungewissem Einfluss auf den Blutdruck wie Metamizol ( $n=8)$, Metoclopramid $(n=6)$, Paracetamol $(\mathrm{n}=5)$ und Diazepam $(\mathrm{n}=3)$ im Urin gefunden.

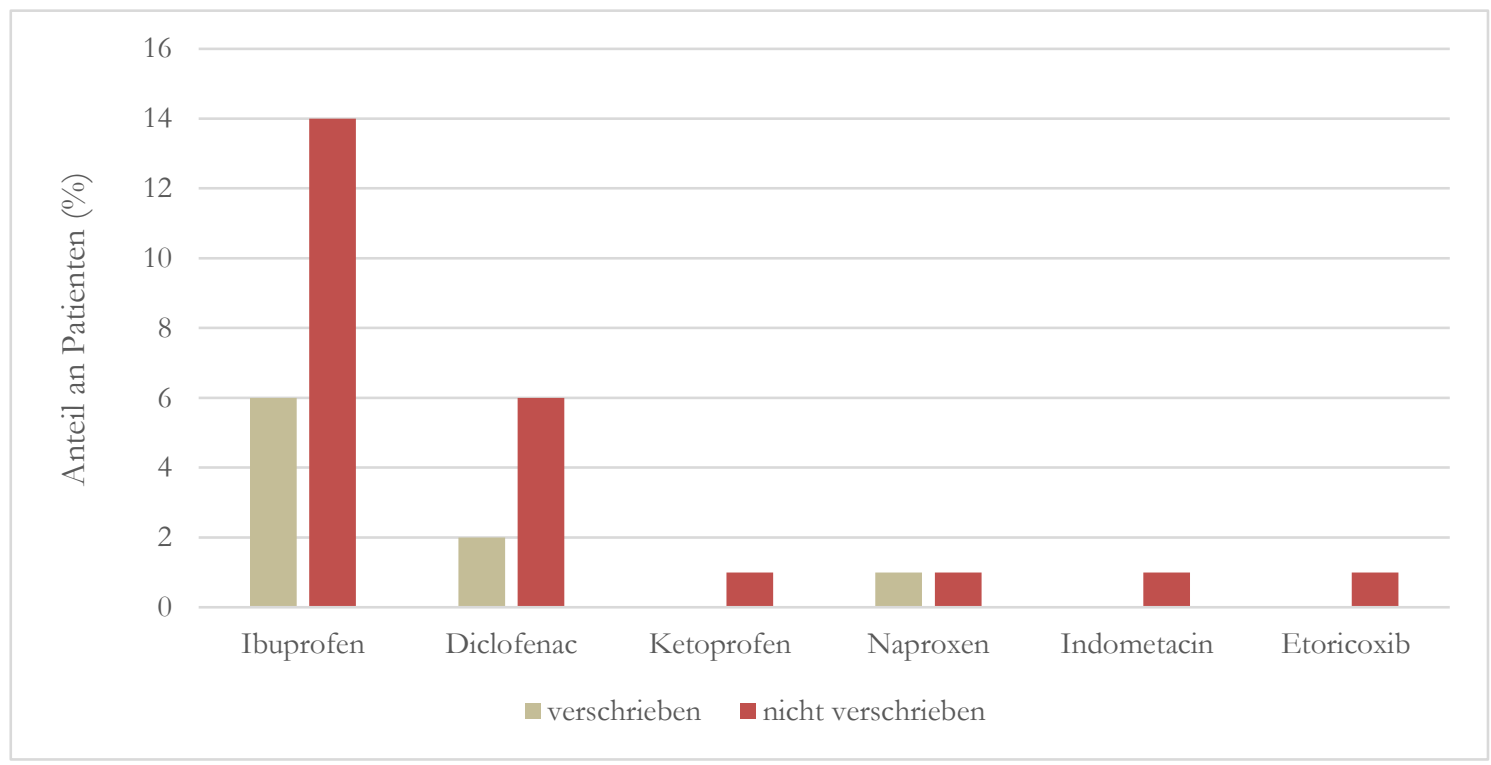

Abbildung 9: Einnahme nichtsteroidaler Antirheumatika insgesamt, $\mathrm{n}=100$.

Betrachtet man die Einnahme von NSAIDs insgesamt, so erhielten 9 Patienten eine Verschreibung und 24 Patienten setzten die Medikamente eigenständig ein. Am häufigsten wurden die frei verkäuflichen Schmerzmittel Ibuprofen und Diclofenac verwendet (Abbildung 9). 


\section{Diskussion}

Frühere retrospektive Studien (Bender et al. 2006; Wu et al. 2010) sowie eine prospektive Studie (Saguner et al. 2010) liefern, unter Verwendung von indirekten Methoden zur Bestimmung der Adhärenz, erste Hinweise darauf, dass die medikamentöse Non-Adhärenz einen wichtigen Risikofaktor für das Auftreten einer HTN-C darstellt. Obwohl es bekannt ist, dass indirekte Methoden zur Bestimmung der Adhärenz, wie die Einschätzung durch Kliniker, Fragebögen, Pillenzählung oder das Einlösen von Rezepten, keine verlässlichen Daten liefern, untersuchte zu Beginn der vorliegenden Arbeit keine dieser Studien die Medikamentenadhärenz mittels einer direkten Methode. Aus diesem Grund konnten alle bisherigen Studien nur sehr störanfällige Daten zur Prävalenz der Non-Adhärenz bei Patienten mit HTN-C liefern. Die vorliegende Studie weist nun erstmals, mittels direkter toxikologischer Bestimmung der Antihypertensiva im Urin, eine außerordentlich hohe Prävalenz (58\%) von Non-Adhärenz bei Patienten mit HTN-C auf. Wenn man bedenkt, dass die Teilnahme an der Adhärenzuntersuchung freiwillig war, könnte man annehmen, dass die Rate der Non-Adhärenz sogar noch höher sein könnte. Bemerkenswerterweise hatten 89\% der non-adhärenten Patienten $\leq 50 \%$ ihrer blutdrucksenkenden Medikamente eingenommen und es bestand eine extrem hohe Diskrepanz zwischen der selbstberichteten und der chemisch-toxikologisch ermittelten Adhärenz.

\subsection{Der prozentuale Anteil an non-adhärenten Patienten}

Der in der vorliegenden Arbeit ermittelte prozentuale Anteil an non-adhärenten Patienten $(58 \%)$ ist höher als in einer kürzlich veröffentlichten Querschnittsstudie mit Patienten mit vorbekannter HTN (28\%, nicht nachweisbare Medikamentenspiegel im Blut), die aufgrund von diversen, auch nicht HTN-assoziierten Ursachen, die Notaufnahme aufsuchten (McNaughton et al. 2017). Die Studie von McNaughton et al. (2017) unterschied jedoch nicht zwischen stabiler HTN und HTN-C bzw. Notfall und schloss Patienten mit meist kontrollierten Blutdruckwerten und einem mittleren systolischen Blutdruck von $137 \pm$ $23 \mathrm{mmHg}$ ein. Im Gegensatz dazu erfüllten in der vorliegenden Stichprobe alle Patienten die Kriterien einer HTN-C und zeigten einen deutlich höheren systolischen Blutdruck von im Mittel $201 \pm 18 \mathrm{mmHg}$. Eine andere ungefähr zeitgleich mit der Publikation der vorliegenden Studie erschienene Adhärenzuntersuchung des Erasmus-Universitätsklinikums in Rotterdam mit Schwerpunkt auf Patienten mit schwerer HTN in der Notaufnahme (ebenfalls als Blutdruck $\geq 180 / 120 \mathrm{mmHg}$ definiert) stellte mithilfe von direkten Adhärenztestungen im Blut eine Non-Adhärenz-Rate von 30,5\% fest. Vier Patienten berichteten spontan von der 
Nicht-Einnahme ihrer Medikamente. Auf der Grundlage der gemessenen Plasmawirkstoffkonzentration wurden $25,5 \%$ der Patienten als non-adhärent eingestuft (mindestens ein verschriebenes blutdrucksenkendes Medikament nicht nachweisbar) (Overgaauw et al. 2019). Mit dem in der niederländischen Studie verwendeten Assay konnten Diuretika und Betablocker, Medikamente, die in der vorliegenden Studie mit einer NonAdhärenz von $43,8 \%$ bzw. 15,4\% verbunden waren, jedoch methodisch bedingt nicht gemessen werden, wodurch die tatsächliche Non-Adhärenz-Rate möglicherweise noch entscheidend unterschätzt wurde.

In der vorliegenden Studie war die Adhärenz im Gesamtkollektiv zu Betablockern und Kalziumantagonisten am höchsten, gefolgt von Angiotensin-II-Blockern, Diuretika, ACEHemmern und Alphablockern. Die erhobenen Adhärenzgrade zu den einzelnen Antihypertensiva differieren teilweise gegenüber den in früheren Studien bei Patienten mit stabiler HTN gefundenen Adhärenzmustern von antihypertensiven Substanzklassen (Kronish et al. 2011; Rizzo und Simons 1997). Hierbei muss jedoch beachtet werden, dass es sich um zwei unterschiedliche Kollektive handelt. Aufgrund der niedrigen Adhärenzrate von Patienten mit HTN-C zu Alphablockern und ACE-Hemmern im vorliegenden Kollektiv, erscheint es vorstellbar, dass Patienten einige Tage vor aufgetretener HTN-C, die entsprechenden Medikamentenklassen abgesetzt bzw. pausiert haben. Ein konsekutiver Rebound-Effekt, durch das akute Pausieren der blutdrucksenkenden Therapie bzw. ein unterschiedliches blutdrucksenkendes Potenzial der einzelnen Substanzklassen der Antihypertensiva, könnte so das Auftreten einer HTN-C begünstigt haben.

\subsection{Stärken und Schwächen der direkten Adhärenzmessung bei Patienten mit HTN-C}

Gegenüber der direkten Adhärenztestung im Blut weist die in der vorliegenden Arbeit erstmals bei Patienten mit HTN-C verwendete Urinuntersuchung mehrere Vorteile auf. Während Blut eine invasive Entnahme und einen genauen Zeitpunkt für die Blutentnahme erfordert, insbesondere bei Arzneimitteln mit hoher Clearance bzw. kurzer Halbwertszeit, bieten Urinproben, durch Anreicherung der Substanz im Urin, ein viel größeres zeitliches Nachweisfenster und den großen Vorteil einer nicht-invasiven Gewinnung.

Ein Patient wird als therapieadhärent eingestuft, wenn das Arzneimittel oder ein Arzneimittelmetabolit in einer durch die Methode noch zuverlässig quantifizierbaren Konzentration vorliegt, und wird umgekehrt als non-adhärent eingestuft, wenn das Arzneimittel oder ein Arzneimittelmetabolit unter seiner Bestimmungsgrenze liegt. 
Bestimmungsgrenzen werden für jedes Molekül als die niedrigstmögliche Konzentration definiert, die mit einer festgelegten Präzision und Genauigkeit gemessen werden kann (Hamdidouche et al. 2017). Die Bestimmungsgrenze ist ein analytischer Parameter, der von der Empfindlichkeit des Massenspektrometers und nicht von der Exposition gegenüber dem Arzneimittel abhängt. Daher kann eine auf direkte toxikologische Adhärenzmessung basierte Definition irreführend sein und einen Patienten fälschlicherweise als adhärent oder nonadhärent einstufen, abhängig nur von der Empfindlichkeit der ChromatographieMassenspektrometrie-Methode (Hamdidouche et al. 2017). Basierend auf bekannten pharmakokinetischen Simulationsdaten scheinen die qualitativen Informationen, die durch einen solchen Ansatz bereitgestellt werden, eine Medikamenten-Non-Adhärenz jedoch in den meisten Fällen zu unterschätzen (Hamdidouche et al. 2017). So ist die in der vorliegenden Arbeit verwendete direkte Bestimmung der Adhärenz nicht geeignet Patienten zu identifizieren, die ihre blutdrucksenkenden Medikamente lediglich einen oder mehrere Tage vor dem medizinischen Besuch einnehmen (White-Coat-Adhärenz). In diesem Fall liegen die Urinkonzentrationen der erwarteten blutdrucksenkenden Medikamente auch über der vordefinierten Bestimmungsgrenze. Demnach werden Patienten mit White-CoatAdhärenz durch die verwendeten Methoden der direkten Adhärenzmessung fälschlicherweise auch als vollständig therapieadhärent eingestuft. Weiterhin ist zu beachten, dass blutdrucksenkende Medikamente (oder ihre Metaboliten) mehrere Tage nach Abbruch der Behandlung im Urin nachgewiesen werden können, insbesondere bei langwirksamen Medikamenten. Beispielsweise dauert es bis zu 27 Tage, bis Amlodipin vollständig im Urin ausgeschieden ist. Daher ist das Risiko, fälschlicherweise auf eine gute Adhärenz während der Tage nach Beendigung der Behandlung zu schließen, hoch (Hamdidouche et al. 2017).

Ein ideales Verfahren zur Überwachung der Adhärenz sollte zuverlässig, praktisch, einfach und relativ kostengünstig sein. Es gibt jedoch keine Methode, die alle diese Kriterien erfüllt (Hamdidouche et al. 2017). Die Auswahl der Methode zur Beurteilung der Medikamentenadhärenz richtet sich nach den Zielen und dem Studienaufbau. In der klinischen Praxis ist zwar bekannt, dass die Selbstberichterstattung von Patienten im Vergleich zu direkten Maßnahmen weniger genau ist, doch kann ihre Verwendung in einem geschäftigen, ressourcenbeschränkten klinischen Umfeld bei einer großen Population mit geringem bis mittelschwerem kardiovaskulären Risiko einen edukativen Wert haben. Regelmäßige Patienteninterviews und die Verwendung von Fragebögen können die Beziehung zwischen Arzt und Patient verbessern. Sie können Ärzten auch dabei helfen, Patienten zu identifizieren, bei denen weitere Beratung erforderlich ist, um die Einhaltung der antihypertensiven Medikation zu fördern (Hamdidouche et al. 2017). Vor dem 
Hintergrund einer hohen Rate an Non-Adhärenz bei Patienten mit scheinbar resistenter HTN erscheint der Einsatz von direkten Methoden zur Adhärenzmessung sinnvoll für die Optimierung des Patientenmanagements (Jung et al. 2013; Kandzari et al. 2018). In klinischen Studien, in denen die Wirksamkeit neuer Therapiestrategien bewertet wird, ist eine genaue Überwachung der Medikamentenadhärenz essenziell (Hamdidouche et al. 2017). Insbesondere gilt dies für die Bewertung von blutdrucksenkenden Behandlungsmethoden. So wurden in neueren Studien zur renalen Denervation explizit direkte Adhärenztestungen durchgeführt, die trotz optimierter Studienbedingungen eine Rate von ca. 40\% an NonAdhärenz aufwiesen (Kandzari et al. 2018).

Unter diesen Bedingungen ist die chemisch-toxikologische Untersuchung auf Antihypertensiva bzw. ihre Metaboliten im Urin eines der besten Instrumente derzeit, um die Therapieadhärenz zu überprüfen. Auch elektronische Überwachungsgeräte können eine wertvolle Hilfe sein. Ein solches Gerät kann jedoch einige Verhaltensverzerrungen hervorrufen, z. B. eine erhöhte Adhärenz, und als solches sind ihre Ergebnisse möglicherweise nicht auf die allgemeine Praxis übertragbar. Wenn für epidemiologische Studien die Beurteilung der Medikamentenadhärenz in Betracht gezogen wird, können die Apothekenaufzeichnungen eine gute Schätzung der Medikamenten-Non-Adhärenz liefern. Schließlich würde eine Kombination aus verschiedenen Methoden, was jedoch im Rahmen der Routine-Patientenversorgung aufgrund des Aufwandes als unpraktikabel einzustufen ist, die validesten Daten zum Einnahmeverhalten liefern, da hierdurch verschiedene Komponenten der Non-Adhärenz identifiziert werden könnten (Hamdidouche et al. 2017).

\subsection{Prädiktoren, die auf das Vorliegen einer Non-Adhärenz in diesem Kollektiv hindeuten}

In Anbetracht der Häufigkeit der HTN-C in der Notaufnahme und der Kosten für die direkte Adhärenzmessung ist es von besonderem Interesse, Patienten mit einem Risiko für NonAdhärenz und der Entwicklung einer HTN-C zu identifizieren. Die vorliegenden Daten legen nahe, dass Begleiterkrankungen wie Diabetes mellitus, Depressionen, chronische Nierenerkrankungen und Herzinsuffizienz mit Non-Adhärenz zur antihypertensiven Medikation bei Patienten mit HTN-C assoziiert sind, ebenso wie eine höhere Anzahl der blutdrucksenkenden Medikamente pro Tag und eine langjährige HTN-Vorgeschichte. Auf der Basis der vorliegenden Daten erscheint es sinnvoll, Patienten mit diesen Merkmalen für ein Adhärenzscreening sowie eine Non-Adhärenzberatung vorzusehen. 
In der logistischen Regression zeigte sich, dass die Wahrscheinlichkeit non-adhärent zu sein bei steigender Anzahl der blutdrucksenkenden Medikamente zunimmt. Dieser Zusammenhang von Komplexität des Therapieschemas und geringer Adhärenz ist im Einklang mit früheren Studien (Claxton et al. 2001; Gupta und Patel und Strauch und Lai und Akbarov und Maresova et al. 2017; Kamran et al. 2014). Diese Tatsache wird durch die aktuellen ESC/ESH-Leitlinien stark hervorgehoben, indem für die meisten Patienten mit HTN eindeutig eine Vereinfachung der Therapie mit Verschreibung einer Einzeltablette zur Behandlung der HTN empfohlen wird (Williams et al. 2018).

Auch Overgaauw et al. (2019) stellten bei ihrer Untersuchung der Unterschiede zwischen adhärenten und non-adhärenten Patienten fest, dass non-adhärenten Patienten mehr blutdrucksenkende Medikamente verschrieben wurden als adhärenten Patienten.

\subsection{Hoher Anteil an potenziell blutdrucksteigernden Medikamenten bzw. Nahrungsmitteln bei Patienten mit HTN-C}

Zusätzlich zur Non-Adhärenz konnte, in bemerkenswertem Ausmaß, die Einnahme von Medikamenten, Nahrungsbestandteilen und/oder weiteren Stoffen, die im Verdacht stehen, an der Entwicklung einer HTN-C beteiligt zu sein, nachgewiesen werden. Zwei in unserem Kollektiv häufig nachgewiesene Medikamentenklassen (NSAIDs und Glucocorticoide) können den Blutdruck durch Volumenretention erhöhen (Lovell und Ernst 2017). In Anbetracht der Tatsache, dass 86\% der eingeschlossenen Patienten eine etablierte blutdrucksenkende Therapie erhielten und 73\% der nachgewiesenen NSAIDs rezeptfreie Medikamente waren, wird die Bedeutung der Aufdeckung einer gleichzeitigen NSAIDBehandlung durch direkte Methoden bei HTN-C ersichtlich.

Außerdem sind Psychopharmaka, die in unserem Kollektiv 10\% der Patienten mit HTN-C eingenommen haben, bekannte Auslöser einer klinisch relevanten Blutdrucksteigerung (Freudenmann et al. 2017; Licht et al. 2009). Für zwei bei unseren Patienten nachgewiesene Psychopharmaka (Citalopram, Venlafaxin) wurde das HTN-Risiko als häufig ( $\geq 1 / 100$ bis $<1 / 10$ ) eingestuft, für drei (Amitriptylin, Nortriptylin, Sertralin) als gelegentlich $(\geq 1 / 1000$ bis $<1 / 100$ ) und für drei (Doxepin, Fluoxetin, Mirtazapin) als unbekannt (Freudenmann et al. 2017). Daher kann nicht ausgeschlossen werden, dass diese Begleitmedikation eine potenzielle Rolle bei der Entwicklung der HTN-C gespielt hat.

In Bezug auf Nahrungs- und Genussmittel waren zwei nachgewiesene Substanzen besonders bemerkenswert. Die Einnahme von Lakritze gaben 10\% der eingeschlossenen Patienten an, während Coffein bei 92\% der Patienten im Urin nachgewiesen werden konnte. Für beide 
Komponenten wurde ein blutdrucksteigernder Effekt beschrieben (Mesas et al. 2011; Penninkilampi et al. 2017).

Ein statistisch signifikanter Anstieg des mittleren systolischen Blutdruckes um $5 \mathrm{mmHg}$ und des diastolischen Blutdruckes um $3 \mathrm{mmHg}$ konnte nach chronischer Einnahme eines Produktes, das Glycyrrhizinsäure enthielt, nachgewiesen werden (Penninkilampi et al. 2017). Auch für das Auftreten einer HTN-C nach der Einnahme von Lakritze finden sich in der Literatur zwei Fallberichte (Giner Galvan et al. 2008; Ottenbacher und Blehm 2015). Coffein kann den Blutdruck durch mehrere Mechanismen erhöhen, einschließlich der Zunahme der Aktivität des sympathischen Nervensystems und Antagonisierung von endogenem Adenosin (Grossman und Messerli 2008). In einer Metaanalyse hatten Patienten, die 200-300 mg Coffein konsumierten, einen durchschnittlichen Anstieg des systolischen und diastolischen Blutdruckes von +8 bzw. +6 mmHg (Mesas et al. 2011). Dieser Anstieg wurde in der ersten Stunde nach der Coffeinaufnahme beobachtet und dauerte $\geq 3$ Stunden. Allerdings führte der regelmäßige Konsum von Kaffee offensichtlich nicht zu einem Anstieg des Blutdruckes (Lovell und Ernst 2017). Da die Konsumgewohnheiten unserer Patienten in Bezug auf Coffein in unserer Studie nicht erfasst wurden, sollten diese Daten daher mit Vorsicht interpretiert werden. Trotz fehlender Unterschiede im Rauchverhalten zwischen adhärenten und non-adhärenten Patienten war der Nachweis von Nikotin im Urin mit einer Prävalenz von $20 \%$ als häufig zu werten. Dies ist von besonderem Interesse, weil Rauchen möglicherweise den Blutdruck erhöht und ein negatives Überleben-Ergebnis mit hypertensiven Notfällen und Rauchen assoziiert ist (Salagre et al. 2017; Virdis et al. 2010).

\subsection{Methodendiskussion}

Aufgrund der bekannten hohen Prävalenz von Non-Adhärenz bei Patienten mit HTN, der Häufigkeit einer HTN-C als Krankheitsbild in der Notaufnahme sowie der vermuteten NonAdhärenz als ein auslösender Faktor für das Auftreten einer HTN-C, stellt die in der vorliegenden Arbeit untersuchte Gruppe von Patienten mit HTN-C, ein relevantes und geeignetes Kollektiv dar, in dem die direkte Analyse der Therapieadhärenz sowie von potenziell blutdrucksteigernden Stoffen durchgeführt wurde.

Mit der direkten toxikologischen Bestimmung der Adhärenz mittels Urinanalysen wurde ein objektives Verfahren verwendet, um die Medikamenten- bzw. Metaboliten-Spiegel direkt zu messen. Unter der Gesamtheit der von allen Patienten eingenommenen blutdrucksenkenden Medikamente konnten durch die GC-MS 23 verschriebene blutdrucksenkende Medikamente nachgewiesen werden. Einschränkungen der Methode im Zusammenhang mit 
antihypertensiven Substanzen betrafen den Nachweis von Nebivolol, Carvedilol, Aliskiren oder Moxonidin, die nur in hohen Konzentrationen nachweisbar waren. Lisinopril, Telmisartan, Candesartan, Doxazosin und Isosorbiddinitrat konnten durch die Methode nicht erfasst werden. Zur Klassifizierung der Patienten in adhärent und non-adhärent wurden nur die eindeutig nachweisbaren blutdrucksenkenden Medikamente herangezogen. Trotzdem liefert diese Technik keine exakten Informationen zum Zeitpunkt oder zur Dosierung der Medikamenteneinnahme, was eine wesentliche Einschränkung unserer Studie ist. Wir können nicht ausschließen, dass einzelne Dosen nicht (reduzierte Persistenz) oder nur in reduzierter Menge (reduzierte Ausführungsqualität) eingenommen wurden. So könnte die Anzahl der vollständig adhärenten Patienten durch die in unserer Studie angewandte Methode sogar überschätzt werden. Darüber hinaus ist zu beachten, dass insbesondere langwirksame Medikamente mehrere Tage nach Abbruch der Behandlung im Urin nachweisbar sind. Daher kann es sein, dass ein Patient fälschlicherweise als vollständig adhärent eingestuft wurde, obwohl er die Einnahme des Medikamentes schon seit Tagen beendet hat. Ein weiterer wesentlicher Aspekt, der bei der Interpretation der vorliegenden Studienergebnisse berücksichtigt werden sollte, ist die relativ kleine Kohorte und die Freiwilligkeit der Teilnahme an der Studie. Die Medikamentenspiegel wurden abhängig von der Einholung einer Einwilligung nach Aufklärung bestimmt, wodurch ein möglicher Selektionsbias der Patienten nicht ausgeschlossen werden kann. Eine Verzerrung der Ergebnisse durch die sogenannte White-Coat-Adhärenz ist ebenfalls denkbar (Cramer et al. 1990; Feinstein 1990). Theoretisch hätten die Patienten ihre blutdrucksenkenden Medikamente kurz vor dem Besuch der Notaufnahme einnehmen können. Dies ist jedoch bei regulären Besuchen in der Klinik möglicherweise häufiger der Fall als in der Notaufnahme (Overgaauw et al. 2019). Eine weitere Einschränkung ist auf das klinische Setting zurückzuführen, in dem die Notfalltherapie zur Verabreichung von blutdrucksenkenden Medikamenten gemäß dem regulären, patientenindividuellen Medikamentenplan führte, wodurch eine zuverlässige Beurteilung der Adhärenz bei mehreren Patienten nicht möglich war. Außerdem ist es nicht auszuschließen, dass einige non-adhärente Patienten ihre Medikamente eingenommen haben, als sie zum ersten Mal Symptome der HTN-C erfuhren. Die in der Studie beobachtete sehr hohe Rate der NonAdhärenz, könnte tatsächlich durch die vorgenannten Aspekte sogar noch wesentlich höher liegen. In dieser Hinsicht gab es eine bemerkenswert hohe Diskrepanz zwischen der selbstberichteten und der chemisch-toxikologisch bewerteten Adhärenz, wobei berücksichtigt werden muss, dass Patienten in der Notaufnahme, die sich mit HTN-C vorstellen, zur Zeit der Vorstellung stark vom Gesundheitssystem abhängig sind. Dies 
könnte die Patienten dazu veranlasst haben, aufgrund der gesellschaftlichen und ärztlichen Erwartungshaltung, einen höheren Grad an Adhärenz anzugeben. Zusätzlich könnten Scham und Schuldgefühle in Bezug auf die möglicherweise selbst verursachte Blutdruckerhöhung durch Nicht-Einnahme der Medikamente das Antwortverhalten der Patienten beeinflusst haben. Schließlich stellt die Art der Blutdruckmessung und Datenerhebung in der Notfallsituation eine mögliche Quelle für Fehler und Verzerrungen der Ergebnisse der vorliegenden Studie dar.

Neue Daten zeigen, dass der Einsatz einer toxikologischen Adhärenztestung zu einer Verbesserung der Adhärenz sowie einem signifikanten Blutdruckabfall bei Patienten mit HTN beitragen kann (Gupta und Patel und Strauch und Lai und Akbarov und Gulsin et al. 2017). Unter Berücksichtigung dieses Aspektes erscheint es denkbar, dass die in der vorliegenden Arbeit verwendete direkte Adhärenztestung auch als Teil eines therapeutischen Ansatzes bei den als non-adhärent eingestuften Patienten mit HTN-C betrachtet werden könnte. Ob die direkte Adhärenztestung in dem vorliegenden Kollektiv zu einer Änderung der Adhärenz geführt hat, wird in einem Anschlussprojekt evaluiert werden.

\subsection{Ausblick}

Trotz einer Reihe neu identifizierter Mechanismen, die in der Pathophysiologie der HTN involviert sind, werden in den nächsten Jahren vermutlich keine wesentlichen pharmakologischen Neuerungen auf dem Markt erwartet (Oparil und Schmieder 2015). Eine eingeschränkte Adhärenz zu den bisher verfügbaren Antihypertensiva ist dabei mit einem erhöhten kardiovaskulären Risiko assoziiert (Burnier et al. 2013; Corrao et al. 2011; Tiffe et al. 2017). So erscheint es äußerst sinnvoll, dass die aktuellen internationalen HTN-Leitlinien, den Stellenwert der Adhärenz und deren Förderung in besonderem Maße hervorheben (Whelton et al. 2018; Williams et al. 2018). Als mögliche Interventionen zur Steigerung einer langfristigen Adhärenz werden integrative Versorgungsmodelle vorgeschlagen, bei denen Hausärzte, Apotheker, Pflegekräfte und spezialisierte Hypertonie-Zentren gemeinsam mit verschiedenen Instrumenten zur Steigerung der Adhärenz beitragen (Williams et al. 2018). Darüber hinaus erscheinen sogenannte Blended-Care-Modelle unter Einbeziehung von Online-Medien, Smartphones und telemedizinischen Strukturen als hoffnungsvolle Ansätze zur Steigerung der Adhärenz (Nerenberg et al. 2018).

Aufgrund der hohen Rate an Non-Adhärenz und Einnahme von blutdrucksteigernden Substanzen bei Patienten mit HTN-C, erscheint es sinnvoll, dass Ärzte in der Notaufnahme die Medikation in Fällen einer HTN-C mit vorbestehender antihypertensiver Medikation 
nicht automatisch eskalieren. Das Wissen um die hohe Prävalenz von Non-Adhärenz sowie der häufige Konsum von blutdrucksteigernden Medikamenten und Nahrungsmitteln sollte ein wesentlicher Bestandteil der Therapieentscheidung sein, um das Wiederauftreten einer HTN-C mit einer effektiven, langfristigen Blutdruckkontrolle sowie einer raschen Blutdrucksenkung zu verhindern. Insbesondere erscheint es sinnvoll, dass Patienten mit HTN-C aufmerksam und aktiv nach Risikofaktoren für Non-Adhärenz befragt werden. Neben kostengünstigen Methoden zur Erhebung der Adhärenz für den Einsatz im klinischen Alltag, könnten im Idealfall direkte Adhärenztestungen bei Patienten mit HTN-C in der Notaufnahme genutzt werden, um die Adhärenz unmittelbar zu bestimmen und eine Therapieempfehlung für eine optimierte Therapie der HTN für Patienten und behandelnden Hausarzt aussprechen zu können. Dies könnte helfen, die wirtschaftlichen Kosten zu senken und zu einer Verringerung der Rehospitalisierungsrate beitragen. Die Integration der direkten Adhärenztestung in den klinischen Routinebetrieb einer Notaufnahme bei Risikopatienten ist jedoch derzeit aufgrund der kosten- und zeitaufwendigen Analytik noch nicht flächendeckend realisierbar.

Es sind größere, fallkontrollierte Studien erforderlich, um die vorliegenden Ergebnisse zu bestätigen und Konzepte zur Steigerung der Therapieadhärenz gemeinsam mit niedergelassenen Hausärzten und betreuenden Fachärzten bei Patienten mit HTN zur Verhinderung einer HTN-C zu evaluieren. 


\section{$5 \quad$ Zusammenfassung}

Aufgrund von retrospektiven Daten bzw. indirekten Adhärenzmessungen wird eine eingeschränkte Medikamentenadhärenz als begünstigender Faktor für das Auftreten einer HTN-C vermutet. Daten aus direkten Adhärenzmessungen waren zum Studienbeginn nicht verfügbar. Das Ziel der vorliegenden Studie war es, den Grad der Adhärenz zu den verschriebenen Antihypertensiva sowie die möglichen Wechselwirkungen von Begleitmedikamenten und Nahrungsmitteln mit den verschriebenen Antihypertensiva bei Patienten mit HTN-C mittels einer direkten toxikologischen Urinanalyse zu ermitteln. In die vorliegende Querschnittsstudie wurden 100 Patienten konsekutiv mit HTN-C (systolischer Blutdruck $\geq 180 \mathrm{mmHg}$ und/oder diastolischer Blutdruck $\geq 120 \mathrm{mmHg}$ ), die in der Notaufnahme der Universitätsmedizin Göttingen aufgenommen wurden, eingeschlossen. Es wurde eine toxikologische Urinanalyse auf das Vorhandensein von Antihypertensiva sowie Begleitmedikation bzw. -stoffen unter Verwendung einer GC-gekoppelten MS durchgeführt. Von 100 Patienten wurden 86 (86\%) mit Antihypertensiva behandelt. Bei insgesamt 62 Patienten konnten die Daten der Urinanalysen eindeutig ausgewertet werden. Bei 15 dieser 62 Patienten (24\%) konnte eine vollständige Non-Adhärenz (kein verschriebenes Antihypertensivum nachweisbar) und bei 21 Patienten (34\%) eine teilweise Non-Adhärenz (mindestens ein Antihypertensivum nicht nachweisbar) nachgewiesen werden. Patienten mit Non-Adhärenz oder teilweiser Non-Adhärenz hatten eine längere HTN-Vorgeschichte (15 [5-22] vs. 10 [3-15] Jahre; $\mathrm{p}=$ 0,04), bekamen mehr blutdrucksenkende Medikamente verschrieben (Anzahl 2,8 \pm 1,1 vs. 1,5 \pm 0,7; $\mathrm{p}<0,01$ ) und wiesen eine höhere Gesamtanzahl an Medikamenten auf $(7,1 \pm 3,4$ vs. 3,4 $\pm 1,8 ; \mathrm{p}<0,01)$. Potenzielle blutdrucksteigernde Begleitmedikamente bzw. -stoffe waren häufig nachweisbar: NSAIDs ( $\mathrm{n}=33)$, Glucocorticoide $(\mathrm{n}=8)$, Antidepressiva $(\mathrm{n}=10)$, Coffein $(\mathrm{n}=92)$, Nikotin $(\mathrm{n}=20)$ und Lakritze ( $\mathrm{n}=10$, mittels Befragung erhoben). Non-Adhärenz und teilweise Non-Adhärenz zu den verschriebenen Antihypertensiva könnte eine entscheidende Rolle für das Auftreten einer HTN-C spielen. Es sind jedoch weitere fallkontrollierte Studien erforderlich, um die vorliegenden Ergebnisse zu bestätigen. Die gleichzeitige Einnahme von rezeptfreien Medikamenten wie NSAIDs, aber auch verschriebenen Medikamenten sowie Nahrungsmitteln könnte ebenfalls zu einer kritischen Steigerung des Blutdruckes beitragen. Um das Auftreten einer HTN-C und damit einhergehende Endorganschäden zu verhindern sowie eine adäquate Behandlung beim Patienten mit bereits eingetretener HTN-C einzuleiten, erscheint es sinnvoll, dass behandelnde Ärzte eine eingeschränkte Adhärenz 
sowie potenziell blutdrucksteigernde Begleitmedikation als potenzielle Ursache für die krisenhafte Blutdruckerhöhung in Betracht ziehen. 


\section{$6 \quad$ Anhang}

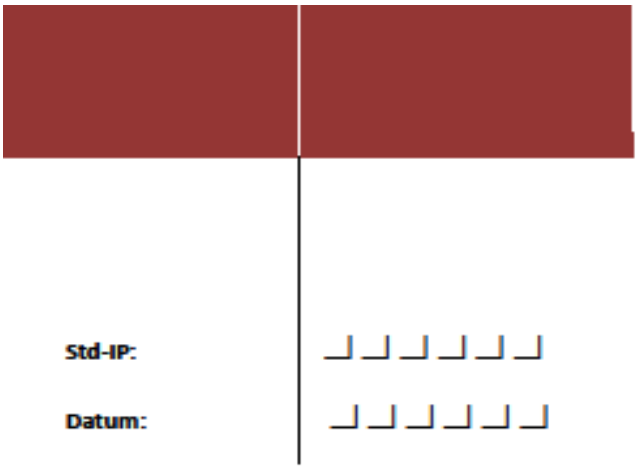

\section{Befragung zu Lebensqualität und krankheitsbezogenen Einstellungen}

\section{SEHR GEEHRTE PATIENTIN, SEHR GEEHRTER PATIENT,}

in unserer Studie geht es uns darum, die Blutdruckbehandlung zu verbessern. Dazu mōchten wir Sie in diesem Fragebogen über Ihren Gesundheitszustand, Ihr persönliches Lebensgefühl sowie Ihre Gedanken, Gefühle und persönlichen Einstellungen zu Bluthochdruck und Ihrer Blutdruckbehandlung befragen. Selbstverständlich ist die Beantwortung freiwillig und ohne Einfluss auf Ihre Behandlung. Alle Ihre Angaben unterliegen der ärtlichen Schweigepflicht und werden nach den Bestimmungen des Datenschutzgesetzes anonym ausgewertet. Eine Weitergabe an Dritte ist ausgeschlossen.

Bitte beachten Sie beim Ausfüllen:

- Bitte beantworten Sie alle Fragen vollständig und so, wie es Ihnen richtig erscheint. Es gibt keine „falschen " Antworten, sondem es geht um lhre persönliche Sicht. Selbstverständlich ist es unbedingt notwendig, dass Sie die Fragen selbst beantworten und sich dabei z.B. nicht „helfen ${ }^{*}$ oder gar „vertreten" lassen. Hilfe ist auch gar nicht möglich, da es bei den Fragen um Ihre ganz persönlichen Empfindungen und Ansichten geht, die nur Sie allein kennen.

- Die Seiten sind sowohl von der Vorderseite als auch von der Rückseite bedruckt. Bitte wenden Sie daher beim Ausfüllen die Seiten!

- Bei den vorliegenden Bögen handelt es sich um abgeschlossene Fragenkomplexe, so dass es sich nicht vermeiden ließ, dass sich einige Fragen ähneln oder wiederholen. Wir bitten Sie dennoch, alle Fragen vollständig mit jeweils einem Kreuz zu beantworten. Sollten Sie bei einer Frage Zweifel haben, kreuzen Sie bitte nur die eine Antwort an, die am ehesten für Sie zutrifft.

- Bei der Beantwortung können Sie sich ruhig Zeit lassen. Allerdings sollten Sie nicht ins Grübeln geraten, sondern eher spontan antworten.

\section{Wir bedanken uns herzlich für Ihre Mitarbeit!}




\section{GESUNDHEITSFRAGEBOGEN SF I 2}

In diesem Fragebogen geht es um Ihre Beurteilung Ihres Gesundheitszustandes. Er ermöglicht, im

Zeitverlauf nachzuvollziehen, wie Sie sich fühlen und im Alltag zurechtkommen.

1. Wie würden Sie Ihren Gesundheitszustand im Allgemeinen beschreiben?

$O$ ausgezeichnet $O$ sehr gut $O$ gut $O$ weniger gut $O$ schlecht

2. Im Folgenden sind einige Tätigkeiten beschrieben, die Sie vielleicht an einem normalen Tag ausūben. Sind Sie durch Ihren derzeitigen Gesundheitszustand bei diesen Tätigkeiten eingeschränkt?

\begin{tabular}{llll}
$\begin{array}{l}\text { Mittelschwere Tätigkeiten, z.B. einen Tisch ver- } \\
\text { schieben, Staubsaugen, Kegeln, Golf spielen }\end{array}$ & Oja, stark & Oja, etwas & Onein, gar nicht \\
\hline Mehrere Treppenabsätze steigen & Oja, stark & Oja, etwas & Onein, gar nicht \\
\hline
\end{tabular}

3. Hatten Sie in den vergangenen $\mathbf{4}$ Wochen aufgrund Ihrer körperlichen Gesundheit irgendwelche Schwierigkeiten bei der Arbeit oder bei anderen alltäglichen Tätigkeiten im Beruf bzw. zu Hause?
Ich habe weniger geschafft als ich wollte
$O$ ja $O$ nein
Ich konnte nur bestimmte Dinge tun
$O$ ja $O$ nein

4. Hatten Sie in den vergangenen $\mathbf{4}$ Wochen aufgrund seelischer Probleme irgendwelche Schwierigkeiten bei der Arbeit oder anderen alltāglichen Tätigkeiten im Beruf bzw. zu Hause (z.B. weil Sie sich niedergeschlagen oder ãngstlich fühlten)?
Ich habe weniger geschafft als ich wollte
O ja O nein
Ich konnte nicht so sorgfalltig wie üblich arbeiten
$O$ ja $O$ nein

5. Inwieweit haben Schmerzen Sie in den vergangenen $\mathbf{4}$ Wochen bei der Ausübung Ihrer Alltagstätigkeiten zu Hause und im Beruf behindert?
O überhaupt nicht
$O$ ein bisschen
O māßig
O ziemlich O sehr

6. In diesen Fragen geht es darum wie Sie sich fühlen und wie es Ihnen in den vergangenen 4 Wochen gegangen ist. Bitte kreuzen Sie in jeder Zeile die Antwort an, die Ihrem Befinden am ehesten entspricht. Wie oft waren Sie in den vergangenen 4 Wochen

\begin{tabular}{lcccccc} 
& immer & meistens & oft & $\begin{array}{c}\text { manch- } \\
\text { mal }\end{array}$ & selten & nie \\
\hline$\ldots$ ruhig und gelassen? & 0 & $\circ$ & 0 & 0 & 0 & 0 \\
\hline$\ldots$ voller Energie? & 0 & $\circ$ & 0 & 0 & 0 & 0 \\
\hline$\ldots$ entmutigt und traurig? & 0 & 0 & 0 & 0 & 0 & 0 \\
\hline
\end{tabular}

7. Wie sehr haben Ihre körperliche Gesundheit oder Ihre seelischen Probleme in den vergangenen 4 Wochen Ihre normalen Kontakte zu Familienangehörigen, Freunden, Nachbarn oder zum Bekanntenkreis beeinträchtigt?
O überhaupt nicht
O etwas
O mäßig
O ziemlich $O$ sehr 


\section{IPQ - KURZFORM}

Wir sind daran interessiert, zu erfahren, was Sie über ihren Bluthochdruck denken. Im Folgenenden finden Sie einige Fragen. Bitte kreuzen Sie diejenige Zahl von 0 bis 10 an, die am besten auf Sie zutrift.

1. Wie stark beeinträchtigt der Bluthochdruck thr Leben?

$\begin{array}{rllllllllllll}\text { gar nicht beeinträchtigt } 0 & 1 & 2 & 3 & 4 & 5 & 6 & 7 & 8 & 9 & 10 & \text { sehr stark beeinträchtigt } \\ & 0 & 0 & 0 & 0 & 0 & 0 & 0 & 0 & 0 & 0 & 0 & \end{array}$

2. Wie lange meinen Sie, werden Sie noch Bluthochdruck haben?

$$
\begin{array}{lllllllllllll}
\text { für immer } & 0 & 1 & 2 & 3 & 4 & 5 & 6 & 7 & 8 & 9 & 10 & \text { nur noch ganz kurz } \\
& 0 & 0 & 0 & 0 & 0 & 0 & 0 & 0 & 0 & 0 & 0 &
\end{array}
$$

3. Wie stark meinen Sie, Ihren Blutdruck selbst durch ihr Verhalten kontrollieren/ beeinflussen zu können?

$\begin{array}{rrrrrrrrrrrrr}\text { absolut keine Kontrolle } & 0 & 1 & 2 & 3 & 4 & 5 & 6 & 7 & 8 & 9 & 10 & \text { extreme Kontrolle } \\ & 0 & 0 & 0 & 0 & 0 & 0 & 0 & 0 & 0 & 0 & 0 & \end{array}$

4. Wie stark meinen Sie, hilft die medikamentöse Behandlung bei Ihrem Bluthochdruck?

$$
\begin{array}{lllllllllllll}
\text { gar nicht } & 0 & 1 & 2 & 3 & 4 & 5 & 6 & 7 & 8 & 9 & 10 & \text { extrem hilfreich } \\
& 0 & 0 & 0 & 0 & 0 & 0 & 0 & 0 & 0 & 0 & 0 &
\end{array}
$$

5. Wie stark spüren Sie Beschwerden durch den Bluthochdruck?

$\begin{array}{lllllllllllll}\text { gar keine Beschwerden } & 0 & 1 & 2 & 3 & 4 & 5 & 6 & 7 & 8 & 9 & 10 & \text { viele starke Beschwerden }\end{array}$ $\begin{array}{lllllllllllllllllll}0 & 0 & 0 & 0 & 0 & 0 & 0 & 0 & 0 & 0 & 0\end{array}$

6. Wie stark sorgen sie sich über Ihren Bluthochdruck?

$$
\begin{array}{lllllllllllll}
\text { gar nicht } & 0 & 1 & 2 & 3 & 4 & 5 & 6 & 7 & 8 & 9 & 10 & \text { extrem } \\
& 0 & 0 & 0 & 0 & 0 & 0 & 0 & 0 & 0 & 0 & 0 &
\end{array}
$$

7. Wie gut meinen Sie, lhren Bluthochdruck zu verstehen?

$$
\begin{array}{lllllllllllll}
\text { gar nicht } & 0 & 1 & 2 & 3 & 4 & 5 & 6 & 7 & 8 & 9 & 10 & \text { sehr gut } \\
& 0 & 0 & 0 & 0 & 0 & 0 & 0 & 0 & 0 & 0 & 0 &
\end{array}
$$

8. Wie stark sind Sie durch Ihren Bluthochdruck gefühlsmäßig beeinträchtigt? (z.B. ärgerlich, verängstigt, aufgewühlt oder niedergeschlagen?)

$\begin{array}{lllllllllllll}\text { gar nicht betroffen } & 0 & 1 & 2 & 3 & 4 & 5 & 6 & 7 & 8 & 9 & 10 & \text { extrem betroffen }\end{array}$

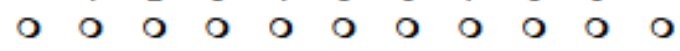

Abbildung A3: IPQ-Kurzform. 


\section{LOT-R / GSE-6}

Bitte geben Sie an, inwieweit lhre Meinung mit den folgenden Aussagen übereinstimmt.

\section{$A 1 / 4$}

1. Auch in ungewissen Zeiten erwarte ich normalerweise das Beste.

2. Es fällt mir leicht, mich zu entspannen.

0 0 o 00

00000

3. Wenn bei mir etwas schief laufen kann, dann tut es das auch.

$0 \quad 0 \quad 0 \quad 0 \quad 0$

4. Meine Zukunft sehe ich immer optimistisch.

0 00000

5. In meinem Freundeskreis fühle ich mich wohl.

$0 \quad 0 \quad 0 \quad 0 \quad 0$

6. Es ist wichtig für mich, ständig beschäftigt zu sein. $\quad 0 \quad 0 \quad \circ \quad \circ \quad$

7. Fast nie entwickeln sich die Dinge nach meinen Vorstellun- 0000000 gen.

8. Ich bin nicht allzu leicht aus der Fassung zu bringen.

00000

9. Ich zähle selten darauf, dass mir etwas Gutes widerfährt.

o 0000

10. Alles in allem erwarte ich, dass mir mehr gute als schlechte Dinge widerfahren

$0 \quad 0 \quad 0 \quad 0 \quad 0$

1. Die Lösung schwieriger Probleme gelingt mir immer, wenn ich mich darum bemühe.

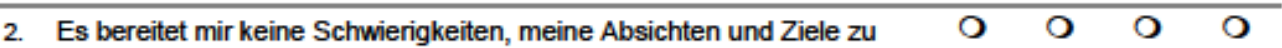
verwirklichen.

3. Auch bei überraschenden Ereignissen glaube ich, dass ich gut damit $\mathrm{O} O \mathrm{O} O \mathrm{O}$ zurechtkommen werde.

\begin{tabular}{llllll}
\hline 4. Was auch immer passiert, ich werde schon klarkommen. & $O$ & $O$ & $O$ & 0
\end{tabular}

5. Wenn ich mit einer neuen Sache konfrontiert werde, weiß ich, wie ich $O \quad O \quad O \quad O$ damit umgehe.

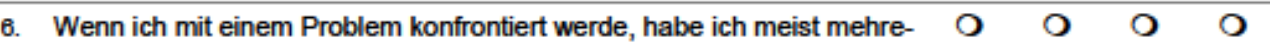
re Ideen, wie ich damit fertig werde. 


\section{HADS}

Bitte beantworten Sie jede der folgenden Fragen so, wie es für Sie persōnlich in der letzten Woche am ehesten zutraf. Machen Sie bitte nur ein Kreuz pro Frage und lassen Sie keine Frage aus! Bitte überlegen Sie nicht lange, wählen Sie die Antwort aus, die Ihnen auf Anhieb am zutreffendsten erscheint!

1. Ich fühle mich angespannt oder überreizt
O meist
O von Zeit zu Zeit
O oft
O überhaupt nicht

2.Ich kann mich heute noch so freuen wie früher

O garz genau so O nicht ganz so sehr $\quad$ O nur noch wenig $/$ gar nicht

3.Mich überkommt eine ängstliche Vorahnung, dass etwas Schreckliches passieren könnte

Oja, sehr stark Oja, abernichtallus stark Oetwes, aber esmadhtmirkeine Oüberhauptricht Sorgen

4.Ich kann lachen und die lustige Seite der Dinge sehen

O ja, so viel wie immer O nicht mehr ganz so viel O inzwischen viel weniger O überhaupt nicht

5.Mir gehen beunruhigende Gedanken durch den Kopf

O einen Großteil der Zeit O verhältrismäßig oft O von Zeitzu Zeit, aber nicht all O nur gelegentlich/nie Zuoft

6.Ich fühle mich glücklich

O überhaupt nicht O selten O manchmal O meistens

7.Ich kann behaglich dasitzen und mich entspannen

O ja, natürlich O gewöhnlich schon O nicht oft

8.Ich fühle mich in meinen Aktivitäten gebremst

O fast immer O sehr oft O manchmal O überhaupt nicht

9.Ich habe manchmal ein ängstliches Gefühl in der Magengegend

O überhaupt nicht O gelegentlich O viemlich oft Osehr oft

10. Ich habe das Interesse an meiner äußeren Erscheinung verloren

Oja, stimmtgenau O ich kümmere mich nichtso O möglicherweise kümmere ich O ich kümmere mich so sehr darum, wie ich sollte mich zu wenig darum viel darum wie immer

11. Ich fühle mich rastlos, muss immer in Bewegung sein

\begin{tabular}{|c|c|c|c|}
\hline Oja, tatsāchlich sehr & O ziemlich & O nicht sehr & Oüberhaupt nicht \\
\hline \multicolumn{4}{|c|}{ 12. Ich blicke mit Freude in die Zukunft } \\
\hline Oja sehr & O eher weniger als früher & O viel weniger als früher & O kaum bis gar nicht \\
\hline
\end{tabular}

\section{Mich überkommt plötzlich ein panikartiger Zustand}
Oja, tatsāchlich sehr oft
O ziemlich oft
O nicht sehr oft
O überhaupt nicht

14. Ich kann mich an einem guten Buch, einer Radio- oder Fernsehsendung freuen
Ooft
O manchmal
O eher selten
O sehr selten

Abbildung A5: HADS. 


\section{ÜBeRZEUgungen Zu MEDIKAMENTEN - BMQ}

Im Folgenden geht es um Ihre Meinung zu Medikamenten. Bitte kreuzen Sie an, wie sehr Sie den folgenden Aussagen zustimmen. Wählen Sie die Antwort aus, die Ihnen auf Anhieb am zutreffendsten erscheint! Machen Sie bitte nur ein Kreuz pro Aussage.

Im ersten Teil geht es ausschließlich um Ihre Blutdruckmedikamente

1. Meine Blutdruckmittel schützen mich davor, dass es mir schlechter geht

2. Meine derzeitige Gesundheit hāngt von meinen Blutdruckmitteln ab $\quad \begin{array}{lllllll}0 & 0 & 0 & 0 & 0\end{array}$

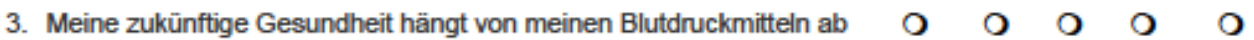

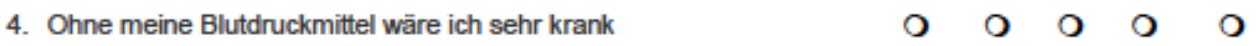

5. Mein Leben, so wie ich es jetzt führe, wäre ohne meine Blutdruck- $\quad 0 \quad 0 \quad 0 \quad 0 \quad 0$ medikamente nicht möglich

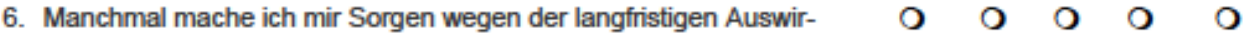
kungen meiner Blutdruckmedikamente

$\begin{array}{llllll}\text { 7. Meine Blutdruckmedikamente sind mir ein Rātsel } & \mathrm{O} & \mathrm{O} & \mathrm{O} & \mathrm{O} & \mathrm{O}\end{array}$
druckmitteln zu werden

9. Es bereitet mir Sorgen, Blutdruckmedikamente nehmen zu müssen $\quad \begin{array}{lllllll}0 & 0 & 0 & 0\end{array}$

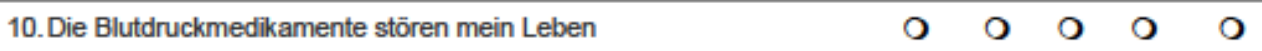

Nun geht es um Medikamente im Allgemeinen

1. Ärzte verwenden zu viele Medikamente $\quad \begin{array}{llllllll} & 0 & 0 & 0 & 0 & 0\end{array}$

2. Wenn Ārzte mehr Zeit für Patienten hätten, würden sie weniger $\quad 0 \quad 0 \quad 0 \quad 0 \quad 0 \quad 0$ Medikamente verschreiben

3. Naturheilmittel sind unbedenklicher als Medikamente $\quad \begin{array}{lllllll}0 & 0 & 0 & 0\end{array}$

4. Die meisten Medikamente machen süchtig $\quad \begin{array}{llllllll} & 0 & 0 & 0 & 0 & 0\end{array}$

5. Menschen, die Medikamente einnehmen, sollten ihre Behandlung $\quad 0 \quad 0 \quad 0 \quad 0 \quad 0$ hin und wieder unterbrechen

\begin{tabular}{|c|c|c|c|c|c|c|}
\hline 6. & Medikamente schaden mehr als dass sie nützen & $\mathrm{O}$ & $\mathrm{O}$ & $\mathrm{O}$ & $\mathrm{O}$ & $\mathrm{O}$ \\
\hline 7. & Alle Medikamente sind Gift & $\mathrm{O}$ & $\mathrm{O}$ & $\mathrm{O}$ & $\mathrm{O}$ & $\mathrm{O}$ \\
\hline
\end{tabular}

Abbildung A6: Überzeugungen zu Medikamenten - BMQ. 


\section{MARS - D / RIEF-INDEX}

Viele Leute nehmen ihre Medikamente so ein bzw. wenden sie so an, wie sie am besten damit zurechtkommen. Dies weicht vielleicht von dem ab, was der Arzt ihnen gesagt hat oder von dem, was im Beipackzettel steht. Wir mōchten gerne von Ihnen erfahren, wie sie selbst lhre aktuellen Medikamente einnehmen / anwenden. Hier finden Sie Aussagen anderer Leute zur Medikamenteneinnahme bzW. anwendung. Bitte kreuzen Sie zu jeder Aussage das Kästchen an, das bei Ihnen am ehesten zutrifft.

Ihre eigene Art, Blutdruckmedikamente einzunehmen / anzuwenden

\begin{tabular}{|c|c|c|c|c|c|}
\hline & immer & oft & $\begin{array}{c}\text { manch- } \\
\text { mal }\end{array}$ & selten & nie \\
\hline ... Ich vergesse sie einzunehmen & $\mathbf{O}$ & 0 & 0 & 0 & $\mathrm{O}$ \\
\hline ... Ich verāndere die Dosis & $\mathcal{O}$ & 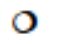 & 0 & 0 & $\mathrm{O}$ \\
\hline ... Ich setze sie eine Weile lang aus & $\mathcal{O}$ & $\mathrm{O}$ & 0 & $\mathrm{O}$ & $\mathrm{O}$ \\
\hline ... Ich lasse bewusst eine Dosis aus & $\mathrm{O}$ & $\mathbf{O}$ & 0 & 0 & $\mathrm{O}$ \\
\hline ... Ich nehme weniger ein / wende weniger an als verordnet & $\mathcal{O}$ & $\mathrm{O}$ & 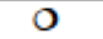 & O & $\mathrm{O}$ \\
\hline
\end{tabular}

Wenn Sie zurückblicken in die Vergangenheit und alle Medikamente Revue passieren lassen, die Ihnen je verschrieben wurden, wie häufig haben Sie folgendes gemacht:

\begin{tabular}{|c|c|c|c|c|c|}
\hline & $\begin{array}{l}\text { fast nie } \\
\text { 0-20\% der } \\
\text { Fäle }\end{array}$ & $\begin{array}{l}\text { sehr selten } \\
20-40 \% \text { der } \\
\text { Fälle }\end{array}$ & $\begin{array}{l}\text { öfters } \\
40-60 \% \\
\text { der Fälle }\end{array}$ & $\begin{array}{l}\text { die meiste } \\
\text { Zeit } \\
60-80 \% \text { der } \\
\text { Fälle }\end{array}$ & $\begin{array}{l}\text { fast immer } \\
80-100 \% \text { der } \\
\text { Fälle }\end{array}$ \\
\hline $\begin{array}{l}\text { Ich habe verschriebene Medikamente noch origi- } \\
\text { nalverpackt gesammelt oder weggeworfen }\end{array}$ & 0 & 0 & 0 & 0 & 0 \\
\hline $\begin{array}{l}\text { Ich habe die Dosierung meiner Medikamente in } \\
\text { Abhängigkeit von meinem Befinden geändert oh- } \\
\text { ne dies mit meinem Arzt zu besprechen }\end{array}$ & 0 & 0 & 0 & 0 & 0 \\
\hline $\begin{array}{l}\text { Ich habe die Medikamenteneinnahme frūher als } \\
\text { von meinem Arzt empfohlen beendet }\end{array}$ & 0 & 0 & 0 & 0 & 0 \\
\hline $\begin{array}{l}\text { Ich habe aufgrund von Neberwirkungen Medika- } \\
\text { mente eigenständig abgesetzt }\end{array}$ & 0 & 0 & 0 & 0 & 0 \\
\hline $\begin{array}{l}\text { Ich habe meine Blutdruckmedikamente eigenstän- } \\
\text { dig abgesetzt, weil mein Blutdruck gut war }\end{array}$ & 0 & 0 & 0 & 0 & $\mathcal{O}$ \\
\hline
\end{tabular}

Abbildung A7: MARS-D/RIEF-Index. 


\section{HERZANGSTFRAGEBOGEN / ESSI}

\begin{tabular}{|c|c|c|c|c|c|}
\hline Bitte kreuzen Sie für jede Aussage Antwort an, die für Sie am besten zutrifft. & nie & selten & $\begin{array}{l}\text { manch- } \\
\text { mal }\end{array}$ & oft & immer \\
\hline 1. Ich beachte aufmerksam meinen Herzschlag. & ○ & ○ & O & ○ & ○ \\
\hline 2. Ich vermeide körperliche Anstrengung. & 0 & 0 & O & 0 & 0 \\
\hline 3. Ich werde nachts durch Herzrasen geweckt. & ○ & ○ & 0 & O & 0 \\
\hline 4. Brustschmerzen / unangenehme Gefühle im Brustbereich wecken mich & 0 & ○ & 0 & 0 & 0 \\
\hline 5. Ich messe meinen Puls. & o & ○ & O & ○ & o \\
\hline 6. Ich vermeide Sport oder körperliche Arbeit. & ○ & ○ & o & ○ & ○ \\
\hline 7. Ich kann mein Herz in meiner Brust spüren. & ○ & ○ & ○ & ○ & 0 \\
\hline 8. Ich vermeide Aktivitäten, die meinen Herzschlag beschleunigen. & ○ & ○ & o & ○ & o \\
\hline $\begin{array}{l}\text { 9. Wenn Untersuchungen normale Ergebnisse erbringen, mache ich mir trotz- } \\
\text { dem Sorgen wegen meines Herzens. }\end{array}$ & o & ○ & o & ○ & O \\
\hline $\begin{array}{l}\text { 10. Ich fühle mich sicher, wenn ich in der Klinik, beim Arzt, oder in einer anderen } \\
\text { medizinischen Einrichtunq bin. }\end{array}$ & 0 & 0 & 0 & ○ & O \\
\hline 11. Ich vermeide Aktivitäten, die mich zum Schwitzen bringen. & o & ○ & ○ & ○ & O \\
\hline 12. Ich befürchte, Ärzte glauben, meine Symptome seien nicht wirklich & ○ & ○ & o & O & 0 \\
\hline
\end{tabular}

Wenn ich unangenehme Gefühle in der Brust habe oder mein Herz schnell schlägt, dann.....

\begin{tabular}{|c|c|c|c|c|c|}
\hline ... Mache ich mir Sorgen, ich könnte eine Herzattacke haben. & o & ○ & ○ & O & o \\
\hline ... Habe ich Schwierigkeiten, mich auf irgendetwas anderes zu konzentrieren. & o & ○ & O & O & o \\
\hline ... Bekomme ich Angst. & ○ & ○ & O & O & ○ \\
\hline ... Möchte ich von einem Arzt untersucht werden. & ○ & o & ○ & O & O \\
\hline ... Spreche ich mit meiner Familie oder Freunden darüber. & ○ & ○ & o & O & O \\
\hline
\end{tabular}

$\begin{aligned} & \text { Die folgenden Fragen beschäftigen sich mit Ihrem Umfeld. Bitte kreuzen Sie zu } \\ & \text { jeder Frage die Antwort an, die Ihre derzeitige Situation am besten beschreibt. }\end{aligned}$
$\begin{aligned} & \text { Wenn Sie sich aussprechen möchten, ist dann jemand für Sie erreichbar, der } \\ & \text { Ihnen mit Gewissheit zuhört? }\end{aligned}$




\section{QUIZ - WIE GUT KENNEN SIE SICH MIT DEM BLUTDRUCK AUS?}

Zum Abschluss wollen wir Ihnen ein paar allgemeine Fragen zum Blutdruck stellen. Es ist immer nur eine Antwort richtig. Wenn Sie die Antwort nicht kennen, raten Sie bitte nicht sondern kreuzen Sie die Antwort „weiß nicht' an.

I. Wofür stehen die beiden Werte bei der Blutdruckmessung?

Für den Blutdruck am Anfang und am Ende der Messung

․ Für den systolischen und diastolischen Blutdruck

․ Für den erhōhten und den optimalen Blutdruck

口 Weiß nicht

2. Welche Aussage zu Beschwerden und Blutdruck stimmt?

․ Bluthochdruck bleibt oft unbemerkt, da er keine Beschwerden verursacht

口 Schon einen Blutdruck von 120 / 80 bemerkt man sofort, da er typische Beschwerden verursacht

․ Eine Blutdruckmessung ist nur bei Beschwerden sinnvoll und nōtig

․ Weiß nicht

3. Welcher Blutdruck ist ${ }_{n}$ gut" ?
口 $100 / 55$
$120 / 80$
ㅁ $160 / 109$
口 Weiß nicht

4. Die Folgen von Bluthochdruck - was ist korrekt?

․ Bluthochdruck ist meistens harmlos, solange er ohne Beschwerden einhergeht

․ Unbehandelter Bluthochdruck kann Blutgefäße schädigen

ㅁ Bluthochdruck kann keinen Herzinfarkt verursachen

口 Weiß nicht

5. Welche Aussagen zum Bluthochdruck sind richtig?

Bluthochdruck ist erblich, da kann man nichts gegen machen

口 Durch Übergewicht wird der Blutdruck gesenkt

口 Regelmāßiger Ausdauersport senkt langfristig den Blutdruck

口 Weiß nicht

6. Welche Aussage über Blutdruckmedikamente ist richtig?

․ Man nimmt sie nur an Tagen mit besonders hohem Blutdruck ( (bei Bedarf") ein

․ Es kōnnen Nebenwirkungen auftreten, die man akzeptieren muss

․ Man sollte regelmāßig pausiert, um zu sehen, ob man sie noch braucht

口. Weiß nicht

\section{Herzlichen Dank für Ihre wertvolle Mitarbeit!}

Abbildung A9: Quiz - Wie gut kennen Sie sich mit dem Blutdruck aus? 


\section{$7 \quad$ Literaturverzeichnis}

Andrade JP, Vilas-Boas F, Chagas H, Andrade M (2002): Epidemiological aspects of adherence to the treatment of hypertension. Arq Bras Cardiol 79, 375-384

Bender SR, Fong MW, Heitz S, Bisognano JD (2006): Characteristics and management of patients presenting to the emergency department with hypertensive urgency. J Clin Hypertens (Greenwich) $\underline{8}, 12-18$

Berra E, Azizi M, Capron A, Hoieggen A, Rabbia F, Kjeldsen SE, Staessen JA, Wallemacq P, Persu A (2016): Evaluation of adherence should become an integral part of assessment of patients with apparently treatment-resistant hypertension. Hypertension 68, 297-306

Burnier M, Wuerzner G, Struijker-Boudier H, Urquhart J (2013): Measuring, analyzing, and managing drug adherence in resistant hypertension. Hypertension $\underline{62}$, 218-225

Chobanian AV, Bakris GL, Black HR, Cushman WC, Green LA, Izzo JL, Jones DW, Materson BJ, Oparil S, Wright JT et al. (2003): Seventh report of the Joint National Committee on Prevention, Detection, Evaluation, and Treatment of High Blood Pressure. Hypertension $\underline{42}$, 1206-1252

Claxton AJ, Cramer J, Pierce C (2001): A systematic review of the associations between dose regimens and medication compliance. Clin Ther $\underline{23}, 1296-1310$

Corrao G, Parodi A, Nicotra F, Zambon A, Merlino L, Cesana G, Mancia G (2011): Better compliance to antihypertensive medications reduces cardiovascular risk. J Hypertens $\underline{29}$, 610618

Corrao G, Parodi A, Zambon A, Heiman F, Filippi A, Cricelli C, Merlino L, Mancia G (2010): Reduced discontinuation of antihypertensive treatment by two-drug combination as first step. Evidence from daily life practice. J Hypertens $\underline{28}$, 1584-1590

Cramer JA, Scheyer RD, Mattson RH (1990): Compliance declines between clinic visits. Arch Intern Med 150, 1509-1510

Feinstein AR (1990): On white-coat effects and the electronic monitoring of compliance. Arch Intern Med 150, 1377-1378

Freudenmann RW, Freudenmann N, Zurowski B, Schonfeldt-Lecuona C, Maier L, Schmieder RE, Lange-Asschenfeldt C, Gahr M (2017): Arterielle Hyper- und Hypotonie assoziiert mit Psychopharmaka: eine Risikobewertung basierend auf den Fachinformationen. Dtsch Med Wochenschr 142, e100-e107

Giner Galvan V, Marco Domingo TF, Martinez Tudela S, Esteban Giner MJ (2008): Hypertensive crisis induced by the ingestion of natural liquorice. Med Clin (Barc) 130, 599

Gonzalez Pacheco H, Morales Victorino N, Nunez Urquiza JP, Altamirano Castillo A, Juarez Herrera U, Arias Mendoza A, Azar Manzur F, Briseno de la Cruz JL, Martinez Sanchez C (2013): Patients with hypertensive crises who are admitted to a coronary care unit: clinical characteristics and outcomes. J Clin Hypertens (Greenwich) 15, 210-214 
Gore JM, Peterson E, Amin A, Anderson FA, Dasta JF, Levy PD, O'Neil BJ, Sung GY, Varon J, Wyman A et al. (2010): Predictors of 90-day readmission among patients with acute severe hypertension. The cross-sectional observational Studying the Treatment of Acute hyperTension (STAT) study. Am Heart J $\underline{160}, 521-527$

Grossman A, Messerli FH, Grossman E (2015): Drug induced hypertension--An unappreciated cause of secondary hypertension. Eur J Pharmacol $\underline{763}, 15-22$

Grossman E, Messerli FH (2008): Secondary hypertension: interfering substances. J Clin Hypertens (Greenwich) 10, 556-566

Grossman E, Messerli FH (2012): Drug-induced hypertension: an unappreciated cause of secondary hypertension. Am J Med 125, 14-22

Gupta AK, Arshad S, Poulter NR (2010): Compliance, safety, and effectiveness of fixed-dose combinations of antihypertensive agents: a meta-analysis. Hypertension $\underline{55}$, 399-407

Gupta P, Patel P, Strauch B, Lai FY, Akbarov A, Gulsin GS, Beech A, Maresova V, Topham PS, Stanley A et al. (2017): Biochemical screening for nonadherence is associated with blood pressure reduction and improvement in adherence. Hypertension $\underline{70}$, 1042-1048

Gupta P, Patel P, Strauch B, Lai FY, Akbarov A, Maresova V, White CMJ, Petrak O, Gulsin GS, Patel V et al. (2017): Risk factors for nonadherence to antihypertensive treatment. Hypertension 69, 1113-1120

Hamdidouche I, Jullien V, Boutouyrie P, Billaud E, Azizi M, Laurent S (2017): Drug adherence in hypertension: from methodological issues to cardiovascular outcomes. J Hypertens $\underline{35}, 1133$ 1144

Herold G: Innere Medizin. Gerd Herold, Köln 2018

Ipek E, Oktay AA, Krim SR (2017): Hypertensive crisis: an update on clinical approach and management. Curr Opin Cardiol $\underline{32}, 397-406$

Janke AT, McNaughton CD, Brody AM, Welch RD, Levy PD (2016): Trends in the incidence of hypertensive emergencies in US emergency departments from 2006 to 2013. J Am Heart Assoc $\underline{5}$, e004511

Jung O, Gechter JL, Wunder C, Paulke A, Bartel C, Geiger H, Toennes SW (2013): Resistant hypertension? Assessment of adherence by toxicological urine analysis. J Hypertens $\underline{31}$, 766774

Kamran A, Sadeghieh Ahari S, Biria M, Malepour A, Heydari H (2014): Determinants of patient's adherence to hypertension medications: application of health belief model among rural patients. Ann Med Health Sci Res 4, 922-927

Kandzari DE, Bohm M, Mahfoud F, Townsend RR, Weber MA, Pocock S, Tsioufis K, Tousoulis D, Choi JW, East C et al. (2018): Effect of renal denervation on blood pressure in the presence of antihypertensive drugs: 6-month efficacy and safety results from the SPYRAL HTN-ON MED proof-of-concept randomised trial. Lancet $\underline{391}$, 2346-2355

Kannel WB (1996): Blood pressure as a cardiovascular risk factor: prevention and treatment. Jama $\underline{275}, 1571-1576$ 
Kronish IM, Woodward M, Sergie Z, Ogedegbe G, Falzon L, Mann DM (2011): Meta-analysis: impact of drug class on adherence to antihypertensives. Circulation $\underline{123}, 1611-1621$

Lewington S, Clarke R, Qizilbash N, Peto R, Collins R (2002): Age-specific relevance of usual blood pressure to vascular mortality: a meta-analysis of individual data for one million adults in 61 prospective studies. Lancet $\underline{360}, 1903-1913$

Licht CM, de Geus EJ, Seldenrijk A, van Hout HP, Zitman FG, van Dyck R, Penninx BW (2009): Depression is associated with decreased blood pressure, but antidepressant use increases the risk for hypertension. Hypertension $\underline{53}$, 631-638

Lovell AR, Ernst ME (2017): Drug-induced hypertension: focus on mechanisms and management. Curr Hypertens Rep 19, 39

Martin JF, Higashiama E, Garcia E, Luizon MR, Cipullo JP (2004): Hypertensive crisis profile. Prevalence and clinical presentation. Arq Bras Cardiol $\underline{83}$, 131-136; 125-130

Maurer HH (2007): Current role of liquid chromatography-mass spectrometry in clinical and forensic toxicology. Anal Bioanal Chem $\underline{388}, 1315-1325$

Maurer HH, Pfleger K, Weber AA (Hrsg.): Mass Spectral and GC Data of Drugs, Poisons, Pesticides, Pollutants, and Their Metabolites. 5. Auflage; Wiley-VCH, Weinheim 2017

McNaughton CD, Brown NJ, Rothman RL, Liu D, Kabagambe EK, Levy PD, Self WH, Storrow AB, Collins SP, Roumie CL (2017): Systolic blood pressure and biochemical assessment of adherence: a cross-sectional analysis in the emergency department. Hypertension $\underline{70}$, 307-314

McNaughton CD, Self WH, Zhu Y, Janke AT, Storrow AB, Levy P (2015): Incidence of hypertension-related emergency department visits in the United States, 2006 to 2012. Am J Cardiol 116, $1717-1723$

Mesas AE, Leon-Munoz LM, Rodriguez-Artalejo F, Lopez-Garcia E (2011): The effect of coffee on blood pressure and cardiovascular disease in hypertensive individuals: a systematic review and meta-analysis. Am J Clin Nutr 쑤, 1113-1126

Nerenberg KA, Zarnke KB, Leung AA, Dasgupta K, Butalia S, McBrien K, Harris KC, Nakhla M, Cloutier L, Gelfer M et al. (2018): Hypertension Canada's 2018 guidelines for diagnosis, risk assessment, prevention, and treatment of hypertension in adults and children. Can J Cardiol $\underline{34}, 506-525$

Neuhauser HK, Adler C, Rosario AS, Diederichs C, Ellert U (2015): Hypertension prevalence, awareness, treatment and control in Germany 1998 and 2008-11. J Hum Hypertens 29, 247253

NIST (Hrsg.): NIST/EPA/NIH MS/MS Mass Spectral Library 2017. John Wiley \& Sons, 2017

Oparil S, Schmieder RE (2015): New approaches in the treatment of hypertension. Circ Res $\underline{116}$, 1074-1095

Ottenbacher R, Blehm J (2015): An unusual case of licorice-induced hypertensive crisis. S D Med $\underline{68}, 346-347,349$ 
Overgaauw N, Alsma J, Brink A, Hameli E, Bahmany S, Peeters LEJ, Van Den Meiracker AH, Schuit SCE, Koch BCP, Versmissen J (2019): Drug nonadherence is a common but often overlooked cause of hypertensive urgency and emergency at the emergency department. J Hypertens $\underline{37}, 1048-1057$

Pandey A, Raza F, Velasco A, Brinker S, Ayers C, Das SR, Morisky DE, Halm EA, Vongpatanasin W (2015): Comparison of Morisky Medication Adherence Scale with therapeutic drug monitoring in apparent treatment-resistant hypertension. J Am Soc Hypertens $\underline{9}$, 420-426

Penninkilampi R, Eslick EM, Eslick GD (2017): The association between consistent licorice ingestion, hypertension and hypokalaemia: a systematic review and meta-analysis. J Hum Hypertens $\underline{31}$, 699-707

Pinna G, Pascale C, Fornengo P, Arras S, Piras C, Panzarasa P, Carmosino G, Franza O, Semeraro V, Lenti $S$ et al. (2014): Hospital admissions for hypertensive crisis in the emergency departments: a large multicenter Italian study. PLoS One $\underline{9}$, e93542

Rizzo JA, Simons WR (1997): Variations in compliance among hypertensive patients by drug class: implications for health care costs. Clin Ther 19, 1446-1457; discussion 1424-1425

Rösner P, Junge T, Westphal F, Fritschi G (Hrsg.): Mass Spectra of Designer Drugs. Wiley-VCH, Weinheim 2017

Sabaté E: Adherence to long-term therapies: evidence for action. World Health Organization, Geneva, Switzerland 2003

Saguner AM, Dur S, Perrig M, Schiemann U, Stuck AE, Burgi U, Erne P, Schoenenberger AW (2010): Risk factors promoting hypertensive crises: evidence from a longitudinal study. Am J Hypertens $\underline{23}, 775-780$

Salagre SB, Itolikar SM, Gedam K (2017): A prospective, observational study to determine the prevalence and clinical profile of patients of hypertensive crisis in a tertiary care hospital. J Assoc Physicians India 65, 14-21

Schmieder RE, Ott C, Schmid A, Friedrich S, Kistner I, Ditting T, Veelken R, Uder M, Toennes SW (2016): Adherence to antihypertensive medication in treatment-resistant hypertension undergoing renal denervation. J Am Heart Assoc 5, e002343

Schönborn P: Methode und Qualität der Adhärenzmessung in randomisiert kontrollierten Studien: Systematic Review. Med. Diss. Göttingen 2009

Tiffe T, Wagner M, Rucker V, Morbach C, Gelbrich G, Stork S, Heuschmann PU (2017): Control of cardiovascular risk factors and its determinants in the general population- findings from the STAAB cohort study. BMC Cardiovasc Disord 17, 276

Tomaszewski M, White C, Patel P, Masca N, Damani R, Hepworth J, Samani NJ, Gupta P, Madira W, Stanley A et al. (2014): High rates of non-adherence to antihypertensive treatment revealed by high-performance liquid chromatography-tandem mass spectrometry (HP LC-MS/MS) urine analysis. Heart $\underline{100}, 855-861$

Van Wijk BL, Klungel OH, Heerdink ER, de Boer A (2005): Rate and determinants of 10-year persistence with antihypertensive drugs. J Hypertens $\underline{23}$, 2101-2107 
Varounis C, Katsi V, Nihoyannopoulos P, Lekakis J, Tousoulis D (2016): Cardiovascular hypertensive crisis: recent evidence and review of the literature front. Cardiovasc Med $\underline{3}, 51$

Virdis A, Giannarelli C, Neves MF, Taddei S, Ghiadoni L (2010): Cigarette smoking and hypertension. Curr Pharm Des $\underline{16}$, 2518-2525

Vrijens B, Vincze G, Kristanto P, Urquhart J, Burnier M (2008): Adherence to prescribed antihypertensive drug treatments: longitudinal study of electronically compiled dosing histories. Bmj $\underline{366}$, 1114-1117

Vuylsteke A, Vincent JL, de La Garanderie DP, Anderson FA, Emery L, Wyman A, Rushton-Smith S, Gore JM (2011): Characteristics, practice patterns, and outcomes in patients with acute hypertension: European registry for Studying the Treatment of Acute hyperTension (EuroSTAT). Crit Care 15, R271

Whelton PK, Carey RM, Aronow WS, Casey DE, Jr., Collins KJ, Dennison Himmelfarb C, DePalma SM, Gidding S, Jamerson KA, Jones DW et al. (2018): 2017 ACC/AHA/AAPA/ABC/ACPM/AGS/APhA/ASH/ASPC/NMA/PCNA Guideline for the prevention, detection, evaluation, and management of high blood pressure in adults: a report of the American College of Cardiology/American Heart Association Task Force on Clinical Practice Guidelines. J Am Coll Cardiol 1ㅡ, e127-e248

Williams B, Mancia G, Spiering W, Agabiti Rosei E, Azizi M, Burnier M, Clement DL, Coca A, de Simone G, Dominiczak A et al. (2018): 2018 ESC/ESH Guidelines for the management of arterial hypertension. Eur Heart J 39, 3021-3104

Wilson SL, Poulter NR (2006): The effect of non-steroidal anti-inflammatory drugs and other commonly used non-narcotic analgesics on blood pressure level in adults. J Hypertens $\underline{24}$, 1457-1469

World Health Organization: The world health report 2002: reducing risks, promoting healthy life. World Health Organization, Geneva, Switzerland 2002

Wu PH, Yang CY, Yao ZL, Lin WZ, Wu LW, Chang CC (2010): Relationship of blood pressure control and hospitalization risk to medication adherence among patients with hypertension in Taiwan. Am J Hypertens 23, 155-160

Zampaglione B, Pascale C, Marchisio M, Cavallo-Perin P (1996): Hypertensive urgencies and emergencies. Prevalence and clinical presentation. Hypertension 27, 144-147 


\section{Danksagung}

Prof. Dr. M. Koziolek und PD Dr. Manuel Wallbach danke ich herzlich für das praxisrelevante, aktuelle Thema und die Möglichkeit meine Dissertation in der Klinik für Nephrologie und Rheumatologie anfertigen zu können.

PD Dr. Manuel Wallbach danke ich für die über die gesamte Zeit andauernde Betreuung und zuverlässige Unterstützung, welche wesentlich zur Fertigstellung der vorliegenden Arbeit beigetragen hat.

Meiner Mitdoktorandin Elena Lowin möchte ich für die kollegiale und engagierte Zusammenarbeit herzlich danken.

Stellvertretend für das Toxikologische Labor der Universitätsmedizin Göttingen spreche ich Hartmud Neurath meinen Dank aus für die technische Durchführung der Urinanalysen.

Herzlichen Dank an die Mitarbeiter der interdisziplinären Notaufnahme für die Hilfsbereitschaft bei der Rekrutierung der Patienten. 